Short communication

\title{
Response a chronic effects of PBDE-47: Up-regulations of HSP60 and HSP70 expression in freshwater bivalve Anodonta woodiana
}

\author{
Xichao Xia ${ }^{\text {a, b, c, * }}$, Shipeng Xue ${ }^{c}$, Xiying Wang ${ }^{c}$, Qingyuan Zhang ${ }^{c}$, Chuanfeng Huang ${ }^{c}$,

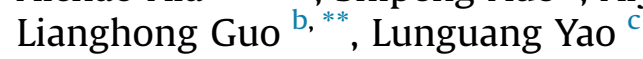 \\ ${ }^{a}$ Medical College of Pingdingshan University, Pingdingshan, 467000, Henan Province, China \\ ${ }^{\mathrm{b}}$ State Key Laboratory of Environmental Chemistry and Eco-toxicology, Research Centre for Eco-environmental Sciences, Chinese Academy of Sciences, \\ Beijing 100085, China \\ ${ }^{\mathrm{c}}$ Department of Basic Medicine, Nanyang Medical College, Nanyang, 473061, Henan Province, China
}

\section{A R T I C L E I N F O}

\section{Article history:}

Received 19 February 2017

Received in revised form

11 April 2017

Accepted 17 April 2017

Available online 19 April 2017

\section{Keywords:}

Anodonta woodiana

PBDE-47

AwHSP60

AwHSP70

\begin{abstract}
A B S T R A C T
Heat shock proteins (HSPs) play an important role in adaption of environmental stress by protein folding, membrane translocation, degradation of misfolded proteins and other regulatory processes. Our previous study showed oxidative stress generated from polybrominated diphenyl ether-47 (PBDE-47) could cause an acute toxicity on freshwater bivalve Anodonta Woodiana, but the effect of chronic toxicity need to be elucidated. In order to further investigate the chronic effect of PBDE-47, clams A. Woodiana were randomly divided into the PBDE-47 treated group administrated with PBDE-47 at a concentration $3.36 \mu \mathrm{g} / \mathrm{L}$ and control group treated with a similar volume dimethyl sulfoxide. Two complete HSP sequences were isolated from A. Woodianaa and respectively named AwHSP60 and AwHSP70. They were widely distributed in foot, gill, hepatopancreas, adductor muscle, heart, hemocytes and mantle. Administration of PBDE-47 could result in a significant up-regulation of AwHSP60 and AwHSP70 expressions in the hepatopancreas, gill and hemocytes. In the hepatopancreas, compared with that of control group, mRNA level of AwHSP60 increased more than $89.9 \%(P<0.05)$ from day $1-15$, AwHSP70 increased more 2.79 times $(P<0.01)$. In the gill, during experiment observed, expression of AwHSP60 increased more 2.09 times $(P<0.01)$ in contrasted with that of control group. Significant up-regulation of AwHSP70 expression showed a reversed U shape. In the hemocytes, AwHSP60 and AwHSP70 expressions of PBDE-47 treated group respectively increased more 2.09 times $(P<0.05)$ and 1.81 times $(P<0.05)$ compared with that of control group. These results indicated that up-regulations of AwHSP60 and AwHSP70 expression are contribute to enhancing adaption of bivalve A. Woodiana exposed to PBDE-47 treatment.
\end{abstract}

() 2017 Published by Elsevier Ltd.

\section{Introduction}

Heat shock proteins (HSPs) are a super-family of proteins which help organisms to modulate stress response and protect organisms from environmental damage [1,2]. Under normal and stress conditions, HSPs function as an important player in protein metabolism, such as protein folding, membrane translocation, degradation of misfolded proteins and other regulatory processes $[3,4]$. According to their molecular weight, HSPs have been divided

\footnotetext{
* Corresponding author. Medical College of Pingdingshan University, Pingdignshan, 467000, Henan Province, China.

** Corresponding author.

E-mail addresses: xiaxichao8336@163.com (X. Xia), lhguo@rcees.ac.cn (L. Guo).
}

into five families including HSP100, HSP90, HSP70, HSP60 and the small HSP20 [5]. Among of them, HSP60 and HSP70 are the most conserved and widely studied proteins [6-9]. HSP70, one conserved protein from bacteria to humans, is best known for its responsiveness to environmental stress $[8,10]$. HSP70 can be induced by various chemicals and biological stresses in order to increase cell's endurance, such as heat shock, oxidative stress, heavy metals, inhibitors of energy metabolism, UV and irradiation, viral and bacterial infections, parasitism, and inflammation [9,11]. HSP60 is involved into the folding of nascent or stress-denatured protein in the steps of binding and encapsulation [12-14].

Bivalves, one group of sedentary filter feeder, have a wide distribution in aquatic environment and are susceptible to various environmental stresses, such as temperature, salinity, and oxygen 
stress, heavy metals and environmental pollutants [15,16]. In order to adapt different environmental conditions, bivalves need develop defense systems that provide protection from harmful environment and potential damage [16]. Characterizations and functions of HSPs have been gain great concern in the process of adaptation. Different sequences of HSPs have been isolated from bivalves. HSP70 have been cloned from various species of bivalves including Crassostrea gigas [17], Crassostrea hongkongensis [18], Ostrea edulis [19], Mytilus galloprovincialis [20], Argopecten irradians [21], Meretrix meretrix [22]. However, to our knowledge, most studies of HSP60 are focused on mammals and typical model organisms, while molecular features and functions of HSP60 in mollusks have seldom been studied. In mollusks, the full length sequences of HSP60 only are found from Aplysia californica, Biomphalaria glabrata, Lottia gigantea nucleotide data and golden apple snail Pomacea canaliculata, but most of them are not yet published [9].

The Chinese pond bivalve Anodonta woodiana, functions as the main criteria required for a bio-indicator organism [23,24]. Earlier studies have revealed the ability of bivalve $A$. woodiana to accumulate trace elements and pesticides, as well as its potential application to detect genotoxicity [25,26]. Meanwhile, these bivalves have important ecosystem functions such as particle filtration and processing, nutrient release, and sediment mixing $[27,28]$. Unfortunately, with exacerbation of environmental deterioration, it has demonstrated that many freshwater mussels have been considered as the most critically endangered groups of animal kingdom [29]. In North America alone, about 36 species are already presumed extinct, and three of eight unionoid species of the North and Central European region are currently listed in the IUCN Red List of Threatened Species [30,31]. Decline of their population should cause serious impact on biological chain as well as freshwater ecosystem.

Polybrominated diphenyl ethers (PBDEs) are common freshwater organic pollutants and have been gained great concern due to their persistence and high bio-accumulative capacity [32]. Among of the different PBDE congeners, PBDE-47 (2,2',4,4'-tetrabromodiphenyl ether), a congener with four bromine atoms, is the most abundant congener in aquatic biota and considered more toxic than the other brominated congeners with respect of overproduction of oxidative stress [33]. Our previous study showed the treatment of PBDE-47 could result in an acute toxicity on calm A. Woodiana, but the effect of chronic toxicity remain unclear [33]. Take into consideration of extensive toxicity of PBDE-47 in aquatic biota, it was of great interest to further investigate the chronic effect of PBDE-47 on bivalve A. Woodiana. In the current study, the complete sequences of HSP70 and HSP60 were cloned from A. Woodiana and respectively named AwHSP70 and AwHSP60, their spatial expressions as well as temporal expressions derived from PBDE-47 exposure were measured by quantitative real-time PCR.

\section{Materials and methods}

\subsection{Ethics statement}

All handling methods of clams were conducted in accordance with the guidelines on the care and use of animals for scientific purposes set up by the Institutional Animal Care and Use Committee of Nanyang Medicine College, Nanyang, China.

\subsection{Materials}

The approximately 1-year-old of clams A. woodiana (shell length, $6.5 \pm 0.5 \mathrm{~cm}$ ) were obtained from the Baihe River of Nanyang, Henan Province, China. Prior to experiment, animals were maintained in a recirculation system containing filtered freshwater at $24{ }^{\circ} \mathrm{C}$ for 2 weeks in laboratory. PBDE-47 was purchased from Sigma-Aldrich (Sigma-Aldrich, St. Louis, MO) and stock solutions were prepared by dissolution in dimethyl sulfoxide (DMSO). The experiment was conducted in some rectangular plastic boxes (40 cm $\times 25 \mathrm{~cm} ; 10 \mathrm{~cm}$ height). The clams were cultured in $10 \mathrm{~L}$ artificial pond containing $48 \mathrm{mg} \mathrm{NaHCO} 3,33 \mathrm{mg} \mathrm{CaCl} 2 \cdot 2 \mathrm{H}_{2} 0,60 \mathrm{mg}$ $\mathrm{MgSO}_{4} \cdot 7 \mathrm{H} 20$ and $0.5 \mathrm{mg} \mathrm{KCl}$ per $1 \mathrm{~L}$ deionized water, with a $\mathrm{pH}$ of 7.0. In order to determine the tissue distribution of AwHSP70 and AwHSP60, 5 clams derived from same tank were dissected, and several of tissues including foot, gill, hepatopancreas, adductor muscle, heart, hemocytes and mantle were sampled prior to the treatment.

In addition, according to above mentioned way of animal treatment, 80 clams were randomly cultured in 10 boxs per 8 mussels/box, these boxes were divided into control group and PBDE-47 treated group per 5 boxs/group. Clams $A$. Woodiana in the PBDE-47 treated groups were administrated with $3.36 \mu \mathrm{g} / \mathrm{L}$ concentrations of PBDE-47, and those in control group with similar volume DMSO. The concentration of DMSO in the water was not excess $0.03 \%$. Both control group and PBDE-47 treated group, 5 clams of every group were respectively pooled from different tanks and dissected. The hepatopancreas, gill and hemocytes were isolated at $0,1,3,6,9,12$ and $15 \mathrm{~d}$, then immediately frozen in liquid nitrogen and stored at $-80^{\circ} \mathrm{C}$ until total RNA extraction.

\subsection{Total RNA isolation and reverse transcription}

Total RNA was extracted using TRIzol (Invitrogen Life Technologies, USA) according to the manufacturer's protocol. Quality of RNA was monitored by $1.2 \%$ agarose gel electrophoresis and those with complete rRNA bands were selected to produce cDNA. Next, total RNA was treated by DNAse (Takara, China). Last, first-strand cDNA was synthesized using M-MLV First-Strand cDNA synthesis Kit (Takara, China) according to the manufacturer's instructions and used as the template for PCR reaction.

\subsection{Cloning of AwHSP60 and AwHSP70 cDNA sequences}

AwHSP60 cDNA fragment was isolated using two degenerate primers AwHSP61 andAwHSP62 with respected of the conserved domain of HSP60 sequences derived from different species (Table 1). Likely, AwHSP70 cDNA fragment was amplified by two degenerate primers using AwHSP71 and AwHSP72 according to the cytosolic HSP70 sequences (Table 1). PCR products were subcloned into the pMDT-19 (Takara, China), sequenced from both directions (Invitrogen Life Technologies, China). The identified HSP60 and HSP70 partial cDNA sequences were selected to undertake RACEPCR. Highly stringent primers (Table 1 ) designed from the partial cDNA sequences were used to characterize the $5^{\prime}$ and $3^{\prime}$ regions of AwHSP60 and AwHSP70 cDNAs by RACE-PCR (Takara, China) according to the manufacturer's protocol. 5' RACE and 3' RACE PCR products were cloned and three clones were sequenced using the method described above.

\subsection{Sequence and phylogenetic analysis}

HSP sequences were analyzed and compared using the BLAST program with a GenBank database search (www.ncbi.nlm.nih.gov/ blast). The signal peptide was predicted by signal program (http:// www.cbs.dtu.dk/services/SignalP). Prediction of protein domain was carried out with the Simple Modular Architecture Research Tool (http://smart.embl-heidelberg.de/). The multiple sequence alignments of AwHSP70 and AwHSP60 genes were performed using the DANMEN analysis program. Prediction of AwHSP70 and AwHSP60 3-D structures were fulfilled by Swiss-model (http:// 
Table 1

Sequences of PCR primers.

\begin{tabular}{|c|c|c|}
\hline Primer & Sequence $\left(5^{\prime}-3^{\prime}\right)$ & Length of amplification (bp) \\
\hline AwHSP61 & GGAGNCAACAGGNAGAACAC & 270 \\
\hline AwHSP62 & TTGGCNAATCTTNCATTCAGC & \\
\hline AwHSP71 & ACCNAAATGNAGGAAACTGC & 520 \\
\hline AwHSP72 & GTGATGCTNGTGTAGAAATC & \\
\hline $5^{\prime}$ Race Innerprimer & CATGGCTACATGCTGACAGCCTA & \\
\hline $5^{\prime}$ Race Outerprimer & CGCGGATCCACAGCCTACTGATGATCAGTCGATG & \\
\hline AwHSP60-5-1 & GTCTTGTTGCCTTTGCCCTTC & 1050 \\
\hline AwHSP60-5-2 & TCTTCAAATACCGTTCCACCCG & \\
\hline AwHSP70-5-1 & CTGGCTTGTGTGCTTGATGA & 640 \\
\hline AwHSP70-5-2 & CCGCCAACCTTCTTATCCAG & \\
\hline 3'Race Outerprimer & TACCGTCGTTCCACTAGTGATTT & \\
\hline $3^{\prime}$ Race Innerprimer & CGCGGATCCTCCACTAGTGATTTCACTATAGG & \\
\hline AwHSP60-3-1 & CGGGTGGAACGGTATTTGAAGA & 890 \\
\hline AwHSP60-3-2 & GAAGGGCAAAGGCAACAAGAC & \\
\hline AwHSP70-3-1 & CTGGATAAGAAGGTTGGCGG & 1410 \\
\hline AwHSP70-3-2 & TCATCAAGCACACAAGCCAG & \\
\hline AwHSP60-F & СTCTCCTTCGATGTCTTCCAGT & 150 \\
\hline AwHSP60-R & TCTTTTCCACTATGACTGTCGA & \\
\hline AwHSP70-F & GGTGGAGCAGGGGGAATGCCAG & 200 \\
\hline AwHSP70-R & CTGCCAATTGGTTGGCATCCAGCC & \\
\hline$\beta-F$ & САTСССТTGСТССТССААСТАТG & 180 \\
\hline$\beta-R$ & CTGGAAGGTAGAGAGAGAAGCCAAG & \\
\hline
\end{tabular}

swissmodel.expasy.org/). Phylogenetic trees constructed from alignment were generated by the Neighbor-joining method using MEGA5.0 software.

\subsection{Quantification of AwHSP60 and AwHSP70 expression by real- time PCR}

To determine the levels of AwHSP60 and AwHSP70 transcript derived from the hepatopancreas and other tissues, real-time quantitative PCR was performed following the manufacture instruction of SYBR Premix Ex TaqTM (TaKaRa, China). Firstly, $\beta$-actin was selected as internal standard according to our previous studies [33,34]. AwHSP60-F and AwHSP60-R primers as well as AwHSP70-F and AwHSP70-R (Table 1) were respectively designed based on isolated sequences of AwHSP60 and AwHSP70, used to isolate target genes in common PCR instrument, and only one band was detected in the PCR production by agarose gel electrophoresis. PCR products were sequenced and identified as the partial sequence of target genes. Subsequently, real-time PCR was fulfilled using an ABI 7500 Real-Time Detection System (Applied Biosystems, USA). Based on characterizations of solubility curve and standard curve constructed, expression levels of AwHSP60 and AwHSP70 were calculated by $2^{-\triangle \triangle \mathrm{CT}}$. All data were given in terms of relative mRNA expression as means \pm SE. One-way ANOVA test analysis of variance (ANOVA) was performed on all data and $P<0.05$ was considered statistically significant.

\section{Results}

\subsection{Characterizations of AwHSP60 and AwHSP70 cDNAs}

A 1, 940 bp nucleotide sequence representing the cDNA sequence of AwHSP60 was obtained by RACE, and deposited in GenBank under accession number KT923182. The full-length cDNA was consisted of a $5^{\prime}$ untranslated region (UTR) of $69 \mathrm{bp}$, a 3' UTR of $167 \mathrm{bp}$ and an open reading frame (ORF) of $1704 \mathrm{bp}$ encoded a polypeptide of 568 amino acids with an estimated molecular mass of $61.03 \mathrm{kDa}$ and theoretical isoelectric point of 5.91 (Fig. 1). A termination signal (AATAAA) was sited at the positions 2000-2005 in the $3^{\prime}$ UTR (Fig. 1). Amino acid sequence of AwHSP60 had several conserved domains of HSP60 family including of mitochondrial target sequence (MTS) between amino acid 1 to 24 (MYRLPSILRPVLTRHLAPCLSRAY), one signature of HSP60 family of amino acid 428-439 (AAVEEGIVPGGG), three highly conserved ATP/ADP binding sites of amino acid 52-57 (TMGPKG), 74-82 (DGVTVAKGI), 193-210 (RDGVITVKDGKTLHDELEV), $\mathrm{Mg}^{2+}$ binding site at amino acids 107-117 (AGDGTTAATVL) and a terminal typical GGM repeat motif at $3^{\prime}$ terminal. Sequence of the amino acids 213-232 (EGMKFDRGYISPYFINTQKG) was responded to traverse the transition region from intermediate domain to the apical domain (Fig. 1).

A 2, 305 bp nucleotide sequence representing the cDNA sequence of AwHSP70 was obtained by RACE, and deposited in GenBank under accession number KT923183. The full-length cDNA was consisted of a 5' UTR of $67 \mathrm{bp}$, a 3' UTR of $267 \mathrm{bp}$ and an ORF of 1971 bp encoding a polypeptide of 657 amino acids with an estimated molecular mass of $71.57 \mathrm{kDa}$ and theoretical isoelectric point of 5.61 (Fig. 2). AwHSP70 had conserved domains of HSP70 family including three signatures of HSP70 family at residues 7-16 (IDIDLGTTYSLGTTYSCV), 197-210 (IFDLGGGTFDVSIL), and 334-348 (IVLVGGSTRIPKV), and two nuclear localization signals of an ATP/GTP binding site at residues 131-137 (AEAYLGQR) and a putative bipartite nuclear localization signal at residues 246-262 (KRKHKKDISDNKRSVRR) (Fig. 2). In addition, a motif of EEVD at the C-terminus was highly conserved throughout all species (Fig. 2). Presence of consecutive repeats of the tetrapeptide motif GGXP in C-terminal region was another notable feature of AwHSP70 (Fig. 2).

\subsection{Evolutionary relationships of AwHSP60 and AwHSP70}

BLAST analysis revealed that amino acid sequence of AwHSP60 was close with other HSP60 family members. The overall deduced amino acid sequence of AwHSP60 exhibited 97.71\% identity to freshwater bivalve Hyriopsis cumingii (JX885711.1), 80.21\% to gastropoda Biomphalaria glabrata (NP_001298236.1), and 76.95\% to gastropoda Aplysia californica (XP_005097387.1). In addition, the AwHSP60 also showed a high similarity with model organisms, such as $75.74 \%$ with Homo sapiens (NP_002147.2), 76.27\% with Mus musculus (NP_034607.3), 73.91\% with Danio rerio (NP_851847.1) and $71.18 \%$ with Drosophila melanogaster (AAF47998.1).

Amino swquence of AwHSP70 exhibited a significant identity with that of HSP70 family members, $98.63 \%$ identity to freshwater bivalve $H$. Cumingii (AHN82525.1), 88.43\% to B. glabrata 

ATGTTGCAGGGAGTTGATTTGCTAGCAGATGCTGTAGCGGTTACCATGGGACCAAAGGGTCGGAATGTGATACTAGAGCAAAGCTGGGGT 270 $\begin{array}{lllllllllllllllllllllllllllllll}\text { M } & \text { L } & \text { Q } & \text { G } & \text { V } & \text { D } & \text { L } & \text { L } & \text { A } & \text { D } & \text { A } & \text { V } & \text { A } & \text { V } & \text { T } & \text { M } & \text { G } & \text { P } & \text { K } & \text { G } & \text { R } & \text { N } & \text { V } & \text { I } & \text { L } & \text { E } & \text { Q } & \text { S } & \text { W } & G & 67\end{array}$ AGCCCAAAGATTACAAAGGATGGAGTTACTGTAGCCAAAGGCATAGATCTGGCAGACAAGTATCAGAACATTGGAGCCAAGCTAGTACAA 360 $\begin{array}{lllllllllllllllllllllllllllllll}\mathrm{S} & \mathrm{P} & \mathrm{K} & \mathrm{I} & \mathrm{T} & \mathrm{K} & \mathrm{D} & \mathrm{G} & \mathrm{V} & \mathrm{T} & \mathrm{V} & \mathrm{A} & \mathrm{K} & \mathrm{G} & \mathrm{I} & \mathrm{D} & \mathrm{L} & \mathrm{A} & \mathrm{D} & \mathrm{K} & \mathrm{Y} & \mathrm{Q} & \mathrm{N} & \mathrm{I} & \mathrm{G} & \mathrm{A} & \mathrm{K} & \mathrm{L} & \mathrm{V} & \mathrm{Q} & 97\end{array}$ GATGTAGCTAACAGTACCAATGAGGAGGCTGGTGATGGTACAACTGCTGCCACAGTTCTTGCCAGGGCTATTGCCAAGGAGGGTTTCGAA 450

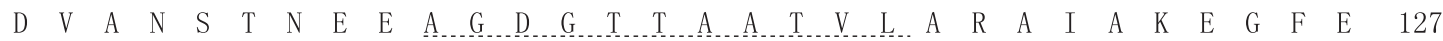
CATATGAGCAGGGGATGTAACCCAATTGAAATCAGGAAAGGTGTAATGATGGCAGTTGATGCTACTGTAGCAGAGTTGAAAAGGATGTCC 540 $\begin{array}{lllllllllllllllllllllllllllllll}\text { H } & \text { M } & \text { S } & \text { R } & G & \text { C } & \text { N } & \text { P } & \text { I } & \text { E } & \text { I } & \text { R } & \text { K } & \text { G } & \text { V } & \text { M } & \text { M } & \text { A } & \text { V } & \text { D } & \text { A } & \text { T } & \text { V } & \text { A } & \text { E } & \text { L } & \text { K } & \text { R } & \text { M } & \text { S } & 157\end{array}$ AAACCAGTTACCACACCTGAGGAAATTGCCCAGGTGGGGACAATTTCAGCTAATGGTGACAAATCTATTGGAGACCTTATCTCGGACGCC 630 $\begin{array}{lllllllllllllllllllllllllllllll}\text { K } & \text { P } & \text { V } & \text { T } & \text { T } & \text { P } & \text { E } & \text { E } & \text { I } & \text { A } & \text { Q } & \text { V } & G & \text { T } & \text { I } & \text { S } & \text { A } & \text { N } & G & \text { D } & \text { K } & \text { S } & \text { I } & \text { G } & \text { D } & \text { L } & \text { I } & \text { S } & \text { D } & \text { A } & 187\end{array}$ ATGAAGAAAGTTGGTAGAGACGGAGTTATCACTGTTAAGGATGGAAAGACGTTACATGATGAATTGGAAGTTATAGAAGGAATGAAGTTT 720 $\begin{array}{lllllllllllllllllllllllllllllll}M & \mathrm{~K} & \mathrm{~K} & \mathrm{~V} & \mathrm{G} & \mathrm{R} & \mathrm{D} & \mathrm{G} & \mathrm{V} & \mathrm{I} & \mathrm{T} & \mathrm{V} & \mathrm{K} & \mathrm{D} & \mathrm{G} & \mathrm{K} & \mathrm{T} & \mathrm{L} & \mathrm{H} & \mathrm{D} & \mathrm{E} & \mathrm{L} & \mathrm{E} & \mathrm{V} & \mathrm{I} & E & G & M & K & F & 217\end{array}$ GACAGGGGCTACATCTCACCTTATTTCATCAATACCCAGAAAGGAGCCAAGTGCGAGTTTCAGGAGGCTCTGGTGCTGTTCTCCGAGAAG 810 $\begin{array}{llllllllllllllllllllllllllllllllllllllll}D & R & G & Y & I & S & P & Y & F & I & N & T & Q & K & G & \mathrm{~A} & \mathrm{~K} & \mathrm{C} & \mathrm{E} & \mathrm{F} & \mathrm{Q} & \mathrm{E} & \mathrm{A} & \mathrm{L} & \mathrm{V} & \mathrm{L} & \mathrm{F} & \mathrm{S} & \mathrm{E} & \mathrm{K} & 247\end{array}$ AAAATCTCCAGTATTCAATCCATCATCCCTGCACTAGAGCTTGCAAATCAGCATCGTAAACCCCTGCTGATCGTGGCTGAGGATGTCGAT 900 $\begin{array}{lllllllllllllllllllllllllllllll}\text { K } & \text { I } & \text { S } & \text { S } & \text { I } & \text { Q } & \text { S } & \text { I } & \text { I } & \text { P } & \text { A } & \text { L } & \text { E } & \text { L } & \text { A } & \text { N } & \text { Q } & \text { H } & \text { R } & \text { K } & \text { P } & \text { L } & \text { L } & \text { I } & \text { V } & \text { A } & \text { E } & \text { D } & \text { V } & \text { D } & 277\end{array}$ GGGGAGGCTCTCACCACCCTTGTTTTAAACAGACTCAAAGTTGGCCTGCAGGTATGTGCAGTGAAAGCCCCAGGCTTCGGAGACAACAGA 990 $\begin{array}{lllllllllllllllllllllllllllllll}\text { G } & \text { E } & \text { A } & \text { L } & \text { T } & \text { T } & \text { L } & \text { V } & \text { L } & \text { N } & \text { R } & \text { L } & \text { K } & \text { V } & \text { G } & \text { L } & \text { Q } & \text { V } & \text { C } & \text { A } & \text { V } & \text { K } & \text { A } & \text { P } & \text { G } & \text { F } & \text { G } & \text { D } & \text { N } & \text { R } & 307\end{array}$ AAGAATACACTCAGGGATATGGCGGTAGCTACGGGTGGAACGGTATTTGAAGATGAAGGGGACATGTTTAAGCTGGAAGACGTTCAACTT 1080 $\begin{array}{lllllllllllllllllllllllllllllll}\text { K } & \text { N } & \text { T } & \text { L } & \text { R } & \text { D } & \text { M } & \text { A } & \text { V } & \text { A } & \text { T } & G & G & \text { T } & \text { V } & \text { F } & \text { E } & \text { D } & \text { E } & \text { G } & \text { D } & \text { M } & \text { F } & \text { K } & \text { L } & \text { E } & \text { D } & \text { V } & \text { Q } & \text { L } & 337\end{array}$ CATGACCTTGGAAAGGTGGGAGAGGTTGCCGTCACCAAGGATGATACTCTGATGATGAAGGGCAAAGGCAACAAGACCGACATTGAAAAA 1170 $\begin{array}{lllllllllllllllllllllllllllllll}\text { H } & \text { D } & \text { L } & G & \text { K } & \text { V } & G & \text { E } & \text { V } & \text { A } & \text { V } & \text { T } & \text { K } & \text { D } & \text { D } & \text { T } & \text { L } & \text { M } & \text { M } & \text { K } & \text { G } & \text { K } & \text { G } & \text { N } & \text { K } & \text { T } & \text { D } & \text { I } & \text { E } & \text { K } & 367\end{array}$ AGACTTCAACAGATCAAAGGTGAGATTGAAACATCGACATCCGATTATAAAAAGGAGAAGCTGAATGAAAGATTAGCTAAATTGTCAAGT 1260 $\begin{array}{lllllllllllllllllllllllllllllll}\mathrm{R} & \mathrm{L} & \mathrm{Q} & \mathrm{Q} & \mathrm{I} & \mathrm{K} & \mathrm{G} & \mathrm{E} & \mathrm{I} & \mathrm{E} & \mathrm{T} & \mathrm{S} & \mathrm{T} & \mathrm{S} & \mathrm{D} & \mathrm{Y} & \mathrm{K} & \mathrm{K} & \mathrm{E} & \mathrm{K} & \mathrm{L} & \mathrm{N} & \mathrm{E} & \mathrm{R} & \mathrm{L} & \mathrm{A} & \mathrm{K} & \mathrm{L} & \mathrm{S} & \mathrm{S} & 397\end{array}$ GGAGTTGCAGTTTTAAAGATTGGAGGAACCAGTGAGGTAGAAGTAAATGAAAAAAAGGATAGAATTAGTGATGCGCTTAACGCTACAAAA 1350 $\begin{array}{llllllllllllllllllllllllllllllll}\text { G } & \text { V } & \text { A } & \text { V } & \text { L } & K & \text { I } & G & G & \text { T } & \text { S } & \text { E } & \text { V } & \text { E } & \text { V } & \text { N } & \text { E } & \text { K } & \text { K } & \text { D } & \text { R } & \text { I } & \text { S } & \text { D } & \text { A } & \text { L } & \text { N } & \text { A } & \text { T } & \text { K } & 427\end{array}$ GCTGCAGTAGAAGAAGGAATTGTACCTGGTGGGGGCACTGCTCTCCTTCGATGTCTTCCAGTGCTAGATACACTTAAACCTGTGAATGAA 1440 \begin{tabular}{|lllllllllllllllllllllllllllllllll}
\hline A & A & V & E & E & $G$ & I & V & P & $G$ & $G$ & $G$ & T & A & L & L & $R$ & C & L & P & V & L & D & T & L & K & P & V & N & E & 457
\end{tabular} GACCAAAGAATAGGTATCGACATAATTAAGAAGGCTTTAAGGGTGCCAGCGTTAACCATCATTAAAAATACTGGATTGGACTCGACAGTC 1530 $\begin{array}{lllllllllllllllllllllllllllllll}\mathrm{D} & \mathrm{Q} & \mathrm{R} & \mathrm{I} & \mathrm{G} & \mathrm{I} & \mathrm{D} & \mathrm{I} & \mathrm{I} & \mathrm{K} & \mathrm{K} & \mathrm{A} & \mathrm{L} & \mathrm{R} & \mathrm{V} & \mathrm{P} & \mathrm{A} & \mathrm{L} & \mathrm{T} & \mathrm{I} & \mathrm{I} & \mathrm{K} & \mathrm{N} & \mathrm{T} & \mathrm{G} & \mathrm{L} & \mathrm{D} & \mathrm{S} & \mathrm{T} & \mathrm{V} & 487\end{array}$ ATAGTGGAAAAGATCTTGATTGAGAAAGGGGAGAGAGGTTACGATGCATTGAATAATGAATTTGTTAATCTCATGGAGAAGGAAATCATC 1620 $\begin{array}{llllllllllllllllllllllllllllllll}\text { I } & \text { V } & \text { E } & \text { K } & \text { I } & \text { L } & \text { I } & \text { E } & \text { K } & G & \text { E } & \text { R } & G & \text { Y } & \text { D } & \text { A } & \text { L } & \text { N } & \text { N } & \text { E } & \text { F } & \text { V } & \text { N } & \text { L } & \text { M } & \text { E } & \text { K } & \text { E } & \text { I } & \text { I } & 517\end{array}$ GATCCAACAAAGGTGGTGCGAACTGCACTAGTGGATGCCTCTGGTGTAGCCTCCCTATTGACAACTGCCGAGTGTGTTGTGACCGAAATC 1710 $\begin{array}{lllllllllllllllllllllllllllllll}\text { D } & \text { P } & \text { T } & \text { K } & \text { V } & \text { V } & \text { R } & \text { T } & \text { A } & \text { L } & \text { V } & \text { D } & \text { A } & \text { S } & G & \text { V } & \text { A } & \text { S } & \text { L } & \text { L } & \text { T } & \text { T } & \text { A } & \text { E } & \text { C } & \text { V } & \text { V } & \text { T } & \text { E } & \text { I } & 547\end{array}$ CCCAAGAAAGAAAGTGCAGGTCCACCAGGAATGGGTGGCATGGGTGGTGGTATGGACATGATGTAGTTGCAGTTCAGACCTGACGTTCCA 1800

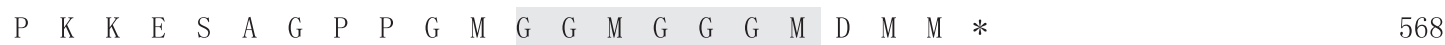
CCACTAAAAGTGCTCAGCACCTTGTTACTACAATACATCTCTCATTTTATTGTTCATTCGTCAAAGAAGATATTGGGACGGACTCGGGAG 1890 TTTGTCATATATAGTAGCAAATAAAGAGCATTGATGCAAAAAAAAAAAAA

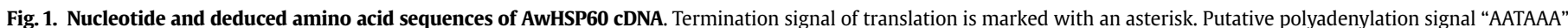

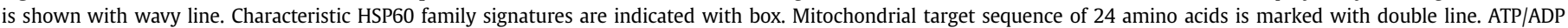

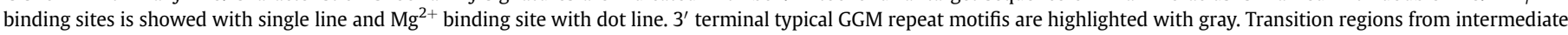
domain to the apical domain are marked with italic script.

(XP_013082114.1) and 87.93\% to Haliotis diversicolor (ACO36047.1). Meanwhile, the AwHSP70 also manifested a high identity with model organism, 86.76\% with M. musculus (AAH66191.1), 85.54\% with D. rerio (NP_001186941.1), 81.76\% with Xenopus laevis
(AAH78115.1), 81.91\% with Daphnia magna (ACB11340.1), 71.88\% D. melanogaster (AAN13545.1).

To examine the relationships of AwHSP60 and AwHSP70, phylogenetic trees were generated by the MEGA 5.0 neighbor 
GAAAGACAAAAGAAAGGAGGCAAGGCAAACACAACCATATCTTATCCAGTTTCAGAACTCTTGGATCATGGCAAAGGTACCAGCTATAGG 90

$\begin{array}{lllllllll}M & A & K & V & P & A & \text { I } & G & 8\end{array}$

AATTGACTTGGGTACTACCTACTCGTGCGTAGGGGTTTTTCAACATGGAAAAGTAGAGATCATTGCCAACGATCAAGGCAACCGAACAAC 180

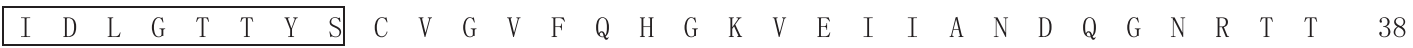

GCCCAGTTATGTTGCCTTCACAGATACAGAGAGGTTAATTGGTGATGCTGCCAAGAACCAGGTGGCTATGAACCCAAACAACACCGTTTT 270

$\begin{array}{llllllllllllllllllllllllllllllll}\mathrm{P} & \mathrm{S} & \mathrm{Y} & \mathrm{V} & \mathrm{A} & \mathrm{F} & \mathrm{T} & \mathrm{D} & \mathrm{T} & \mathrm{E} & \mathrm{R} & \mathrm{L} & \mathrm{I} & \mathrm{G} & \mathrm{D} & \mathrm{A} & \mathrm{A} & \mathrm{K} & \mathrm{N} & \mathrm{Q} & \mathrm{V} & \mathrm{A} & \mathrm{M} & \mathrm{N} & \mathrm{P} & \mathrm{N} & \mathrm{N} & \mathrm{T} & \mathrm{V} & \mathrm{F} & 68\end{array}$

TGATGCCAAACGTTTGATTGGCAGAAAGTTTAATGAACCCTCTGTTCAGTCTGATATGAAGCACTGGCCGTTTGATGTAATCAATGATGG 360

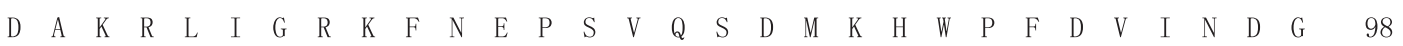

TGGAAAACCCAAGATAAAGGTAGAATACAAAGGAGAGGAGAAAACATTTTATCCTGAAGAAATCTCCTCAATGGTTTTGACCAAAATGAA 450

$\begin{array}{llllllllllllllllllllllllllllllll}\text { G } & K & P & K & I & K & V & E & Y & K & G & E & E & K & T & F & Y & P & E & E & I & S & S & M & V & \text { L } & \text { T } & \text { K } & \text { M } & \text { K } & 128\end{array}$

GGAAACTGCCGAGGCTTATCTTGGAAAGGTCGTTACAAATGCTGTAGTAACTGTGCCAGCATACTTTAACGATTCACAGAGACAGGCCAC 540

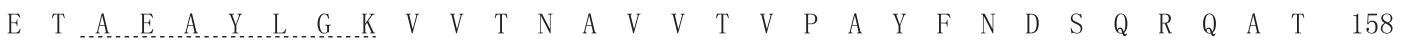
GAAAGATGCTGGAACAATTGCTGGTCTTAATGTACTCCGTATCATCAATGAGCCTACAGCTGCTGCCATTGCCTATGGCCTGGATAAGAA 630 $\begin{array}{lllllllllllllllllllllllllllllll}\text { K } & \text { D } & \text { A } & G & \text { T } & \text { I } & \text { A } & G & \text { L } & \text { N } & \text { V } & \text { L } & \text { R } & \text { I } & \text { I } & \text { N } & \text { E } & \text { P } & \text { T } & \text { A } & \text { A } & \text { A } & \text { I } & \text { A } & \text { Y } & \text { G } & \text { L } & \text { D } & \text { K } & \text { K } & 188\end{array}$ GGTTGGCGGTGAAAAAAATGTCTTGATCTTTGATCTTGGAGGCGGTACTTTTGATGTCTCCATTCTGACCATCGAGGATGGCATTTTTGA 720 $\begin{array}{llllllllllllllllllllllllllllllllll}\mathrm{V} & G & G & \mathrm{E} & \mathrm{K} & \mathrm{N} & \mathrm{V} & \mathrm{L} & \mathrm{I} & \mathrm{F} & \mathrm{D} & \mathrm{L} & \mathrm{G} & \mathrm{G} & \mathrm{G} & \mathrm{T} & \mathrm{F} & \mathrm{D} & \mathrm{V} & \mathrm{S} & \mathrm{I} & \mathrm{L} & \mathrm{T} & \mathrm{I} & \mathrm{E} & \mathrm{D} & \mathrm{G} & \mathrm{I} & \mathrm{F} & \mathrm{E} & 218\end{array}$ AGTTAAATCTACATCTGGAGACACCCATTTGGGTGGAGAGGACTTTGACAACAGAATGGTCAATCACTTCGTCCAGGAATTTAAGAGGAA 810 $\begin{array}{llllllllllllllllllllllllllllllll}\mathrm{V} & \mathrm{K} & \mathrm{S} & \mathrm{T} & \mathrm{S} & \mathrm{G} & \mathrm{D} & \mathrm{T} & \mathrm{H} & \mathrm{L} & \mathrm{G} & \mathrm{G} & \mathrm{E} & \mathrm{D} & \mathrm{F} & \mathrm{D} & \mathrm{N} & \mathrm{R} & \mathrm{M} & \mathrm{V} & \mathrm{N} & \mathrm{H} & \mathrm{F} & \mathrm{V} & \mathrm{Q} & \mathrm{E} & \mathrm{F} & \underline{\mathrm{K}} & \mathrm{R} & \mathrm{K} & 248\end{array}$ ACACAAGAAAGACATCAGTGACAACAAGAGATCAGTTCGTCGATTGAGAACCGCATGTGAAAGAGCCAAGAGGACACTGTCATCAAGCAC 900 \begin{tabular}{lllllllllllllllllllllllllllllll}
$\mathrm{H}$ & $\mathrm{K}$ & $\mathrm{K}$ & $\mathrm{D}$ & $\mathrm{I}$ & $\mathrm{S}$ & $\mathrm{D}$ & $\mathrm{N}$ & $\mathrm{K}$ & $\mathrm{R}$ & $\mathrm{S}$ & $\mathrm{V}$ & $\mathrm{R}$ & $\mathrm{R}$ & $\mathrm{L}$ & $\mathrm{R}$ & $\mathrm{T}$ & $\mathrm{A}$ & $\mathrm{C}$ & $\mathrm{E}$ & $\mathrm{R}$ & $\mathrm{A}$ & $\mathrm{K}$ & $\mathrm{R}$ & $\mathrm{T}$ & $\mathrm{L}$ & $\mathrm{S}$ & $\mathrm{S}$ & $\mathrm{S}$ & $\mathrm{T}$ & 278 \\
\hline
\end{tabular} ACAAGCCAGTGTGGAAATTGATTCACTCTATGAAGGAATTGATTTCTACACCAGCATCACCAGGGCAAGATTTGAAGAACTGAATGCTGA 990 $\begin{array}{llllllllllllllllllllllllllllllll}\text { Q } & \text { A } & \text { S } & \text { V } & \text { E } & \text { I } & \text { D } & \text { S } & \text { L } & \text { Y } & \text { E } & G & \text { I } & \text { D } & \text { F } & \text { Y } & \text { T } & \text { S } & \text { I } & \text { T } & \text { R } & \text { A } & \text { R } & \text { F } & \text { E } & \text { E } & \text { L } & \text { N } & \text { A } & \text { D } & 308\end{array}$ CTTGTTCAGAGGAACTCTTGAACCAGTTAAGAAGGCTCTTCGGGATGCGAAGATGGACAAGCCGCAAGTACATGATATTGTGTTGGTAGG 1080 $\begin{array}{llllllllllllllllllllllllllllllll}\mathrm{L} & \mathrm{F} & \mathrm{R} & \mathrm{G} & \mathrm{T} & \mathrm{L} & \mathrm{E} & \mathrm{P} & \mathrm{V} & \mathrm{K} & \mathrm{K} & \mathrm{A} & \mathrm{L} & \mathrm{R} & \mathrm{D} & \mathrm{A} & \mathrm{K} & \mathrm{M} & \mathrm{D} & \mathrm{K} & \mathrm{P} & \mathrm{Q} & \mathrm{V} & \mathrm{H} & \mathrm{D} & \mathrm{I} & \mathrm{V} & \mathrm{L} & \mathrm{V} & \mathrm{G} & 338\end{array}$ AGGGTCCACTCGTATCCCGAAAGTCCAGGAACTTCTCCAGGATTTCTTCAATGGCAAGGAACTGAACAAGAGCATTAACCCCGACGAGGC 1170 \begin{tabular}{|llllllllllllllllllllllllllllllll}
\hline$G$ & S & T & R & I & P & K & V & Q & E & L & L & Q & D & F & F & N & $G$ & K & E & L & N & K & S & I & N & P & D & E & A & 368
\end{tabular} AGTTGCCTATGGAGCAGCTGTTCAGGCTGCAATTCTTCAGGGCGACAAATCTGAGGCTGTCCAGGACCTTCTGCTGCTCGATGTTGCCCC 1260

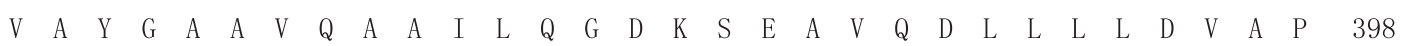
ATTGTCTTTAGGTATTGAGACGGCTGGTGGAGTAATGACTGCCCTGATCAAGAGAAACACCACCATTCCCACCAAACAGACACAGACCTT 1350 $\begin{array}{lllllllllllllllllllllllllllllll}\mathrm{L} & \mathrm{S} & \mathrm{L} & \mathrm{G} & \mathrm{I} & \mathrm{E} & \mathrm{T} & \mathrm{A} & \mathrm{G} & \mathrm{G} & \mathrm{V} & \mathrm{M} & \mathrm{T} & \mathrm{A} & \mathrm{L} & \mathrm{I} & \mathrm{K} & \mathrm{R} & \mathrm{N} & \mathrm{T} & \mathrm{T} & \mathrm{I} & \mathrm{P} & \mathrm{T} & \mathrm{K} & \mathrm{Q} & \mathrm{T} & \mathrm{Q} & \mathrm{T} & \mathrm{F} & 428\end{array}$ TACTACCTACTCCGACAACCAGCCTGGTGTGCTGATCCAGGTCTATGAAGGTGAAAGAGCCATGACCAAAGATAACAACCTCCTTGGTAA 1440 $\begin{array}{lllllllllllllllllllllllllllllll}\mathrm{T} & \mathrm{T} & \mathrm{Y} & \mathrm{S} & \mathrm{D} & \mathrm{N} & \mathrm{Q} & \mathrm{P} & \mathrm{G} & \mathrm{V} & \mathrm{L} & \mathrm{I} & \mathrm{Q} & \mathrm{V} & \mathrm{Y} & \mathrm{E} & \mathrm{G} & \mathrm{E} & \mathrm{R} & \mathrm{A} & \mathrm{M} & \mathrm{T} & \mathrm{K} & \mathrm{D} & \mathrm{N} & \mathrm{N} & \mathrm{L} & \mathrm{L} & \mathrm{G} & \mathrm{K} & 458\end{array}$ ATTTGAGCTTACGGGAATTCCTCCTGCACCACGTGGCGTACCCCAAATTGAGGTGACCTTTGACATAGATGCCAATGGTATTCTCAACGT 1530 $\begin{array}{lllllllllllllllllllllllllllllll}\mathrm{F} & \mathrm{E} & \mathrm{L} & \mathrm{T} & \mathrm{G} & \mathrm{I} & \mathrm{P} & \mathrm{P} & \mathrm{A} & \mathrm{P} & \mathrm{R} & \mathrm{G} & \mathrm{V} & \mathrm{P} & \mathrm{Q} & \mathrm{I} & \mathrm{E} & \mathrm{V} & \mathrm{T} & \mathrm{F} & \mathrm{D} & \mathrm{I} & \mathrm{D} & \mathrm{A} & \mathrm{N} & \mathrm{G} & \mathrm{I} & \mathrm{L} & \mathrm{N} & \mathrm{V} & 488\end{array}$ TTCTGCAGTGGACAAAAGCACTGGCAAAGAAAACAAGATTACTATTACCAATGACAAAGGACGTCTTAGCAAGGATGAAATTGAACGCAT 1620 $\begin{array}{lllllllllllllllllllllllllllllll}\mathrm{S} & \mathrm{A} & \mathrm{V} & \mathrm{D} & \mathrm{K} & \mathrm{S} & \mathrm{T} & \mathrm{G} & \mathrm{K} & \mathrm{E} & \mathrm{N} & \mathrm{K} & \mathrm{I} & \mathrm{T} & \mathrm{I} & \mathrm{T} & \mathrm{N} & \mathrm{D} & \mathrm{K} & \mathrm{G} & \mathrm{R} & \mathrm{L} & \mathrm{S} & \mathrm{K} & \mathrm{D} & \mathrm{E} & \mathrm{I} & \mathrm{E} & \mathrm{R} & \mathrm{M} & 518\end{array}$ GGTGAATGATGCAGAGAAGTACAAAGGTGAAGATTCAAAACAGAGAGACCGTGTTGCTGCCAAGAATTCACTTGAAAGCTATGCATTCCA 1710 $\begin{array}{lllllllllllllllllllllllllllllll}\mathrm{V} & \mathrm{N} & \mathrm{D} & \mathrm{A} & \mathrm{E} & \mathrm{K} & \mathrm{Y} & \mathrm{K} & \mathrm{G} & \mathrm{E} & \mathrm{D} & \mathrm{S} & \mathrm{K} & \mathrm{Q} & \mathrm{R} & \mathrm{D} & \mathrm{R} & \mathrm{V} & \mathrm{A} & \mathrm{A} & \mathrm{K} & \mathrm{N} & \mathrm{S} & \mathrm{L} & \mathrm{E} & \mathrm{S} & \mathrm{Y} & \mathrm{A} & \mathrm{F} & \mathrm{H} & 548\end{array}$ TATGAAATCCACGGTTGAAGACCAGAATCTTAAAGACAAGATAAGTGAGTCTGATAGAAAGATCATCACAGACAAATGCAACGATATCAT 1800 $\begin{array}{lllllllllllllllllllllllllllllll}\text { M } & \text { K } & \text { S } & \text { T } & \text { V } & \text { E } & \text { D } & \text { Q } & \text { N } & \text { L } & \text { K } & \text { D } & \text { K } & \text { I } & \text { S } & \text { E } & \text { S } & \text { D } & \text { R } & \text { K } & \text { I } & \text { I } & \text { T } & \text { D } & \text { K } & \text { C } & \text { N } & \text { D } & \text { I } & \text { I } & 578\end{array}$ TTCATGGCTGGATGCCAACCAATTGGCAGATAAGGAGGAGTTTGAACACAAGCAGAAGGAGATTGAAGGTGTTTGTAATCCTATAATTAC 1890 $\begin{array}{lllllllllllllllllllllllllllllll}\mathrm{S} & \text { W } & \mathrm{L} & \mathrm{D} & \mathrm{A} & \mathrm{N} & \mathrm{Q} & \mathrm{L} & \mathrm{A} & \mathrm{D} & \mathrm{K} & \mathrm{E} & \mathrm{E} & \mathrm{F} & \mathrm{E} & \mathrm{H} & \mathrm{K} & \mathrm{Q} & \mathrm{K} & \mathrm{E} & \mathrm{I} & \mathrm{E} & \mathrm{G} & \mathrm{V} & \mathrm{C} & \mathrm{N} & \mathrm{P} & \mathrm{I} & \mathrm{I} & \mathrm{T} & 608\end{array}$ GAAGCTGTATCAGCAGGCTGGTGGAGCAGGGGGAATGCCAGGAGGTATGCCAGGTGGATTCCCAGGTGGTGCCCCTGGTGCAGGTGGTCA 1980 $\begin{array}{lllllllllllllllllllllllllllllll}K & L & Y & Q & Q & A & G & G & A & G & G & M & P & G & G & M & P & G & G & F & P & G & G & A & P & G & A & G & G & H & 638\end{array}$ TGGTGGCCATGGTCATTCCGCTGGTAGTAGCGGAGGCCCAACTATTGAGGAAGTTGATTAAAAAGATCATTGTAACATGAATCAAACTGT 2070 $\begin{array}{lllllllllllllllllllll}G & G & H & G & H & S & A & G & S & S & G & G & P & T & I & E & E & V & D & *\end{array}$ 657 ATTAGGGATTAAAATAAAAATTCTCCAATTGGAACTGGTTTAGGCACATACAAGTTGTTGTAAAAGTTTTCACATTTTTTTTTGTTTAGT 2160 GCTGCAAAGCATTTTGTTGCCCAACAAGTGTTTACTTCAAAAAGGATGTTCAGTAAAATACTACTGTTTGAATGATAACGTAATTAAAA 2250 AGTTTAATGTTTAGATTCGAAATTGCAATTTTGTTTAATTTGAAAAAAAAAAAAA

2305

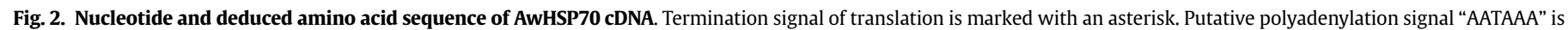

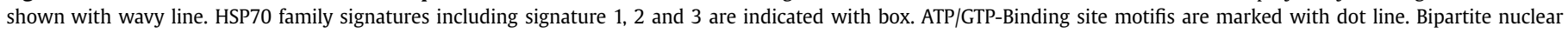
targeting sequences are indicated with single underline. The last four amino acids that form a motif 'EEVD' are marked with shadow. 
joining method using different HSP60 and HSP70 family members selected from vertebrate and invertebrate species, respectively (Figs. 3 and 4). The AwHSP60 was closest to that of bivalve and gastropoda, then fish and mammal, next insect and crustacean, last bacteria (Fig. 3). AwHSP70 was closest to that of bivalve and gastropoda, then insect and crustacean mammal, next vertebrates, last bacteria (Fig. 4). In evidenced sequences of mollusk, AwHSP60 and AwHSP70 showed a most relationship of evolution with calms of freshwater.

\subsection{Tissue distributions of AwHSP60 and AwHSP70 mRNA}

Results of real time PCR showed expressions of AwHSP60 and AwHSP70 were widely distributed in foot, gill, heart, hepatopancreas, hemocytes, adductor muscle and mantle of bivalve A. woodiana (Fig. 5). In selected tissues, the highest expression of AwHSP60 mRNA was observed in the hepatopancreas and gill, intermediate ones in the hemocytes, mantle and heart, lower ones in foot and adductor muscle (Fig. 5A). The mRNA level of AwHSP70 showed a high expression in the hepatopancreas, a moderate level in gill and hemocytes, a lower level in heart, adductor muscle and mantle (Fig. 5B).

\subsection{Temporal expressions of AwHSP70 and AwHSP60 in hepatopancreas, gill and hemocytes exposured to PBDE-47}

In the hepatopancreas, gill and hemoctyes, a stable profile of AwHSP60 and AwHSP70 expression were observed in control group during experiment observed. But, expressions of AwHSP60 and AwHSP70 were significantly affected and showed different characterizations in the PBDE-47 treated group.

In the hepatopancreas, expressions of AwHSP60 and AwHSP70 mRNA were significantly up-regulated by PBDE-47 treatment using RT-qPCR with $\beta$-actin as internal control from day $1-15$ (Fig. 6). Upregulation of AwHSP60 mRNA level showed a time-dependent pattern. mRNA level of AwHSP60 increased more than $89.9 \%$ at day $1(P<0.05)$ and 6.73 times $(P<0.01)$ at day 15 in contrasted with that of control group (Fig. $6 \mathrm{~A}$ ). Compared with that control group, expression of AwHSP70 was significantly induced from day $1-15$, increased more 2.79 times $(P<0.01)$ at day 1 , even to 13.15 times $(P<0.01)$ at day 15 (Fig. $6 \mathrm{~B})$.

In the gill, administration of PBDE-47 could result in a significant effect on expressions of AwHSP60 and AwHSP70 (Fig. 7). After PBDE-47 treatment, expression of AwHSP60 was significantly induced from day $1-15$, increased more 2.09 times $(P<0.01)$ (Fig. 7A). Like profile of AwHSP60, compared with that of control

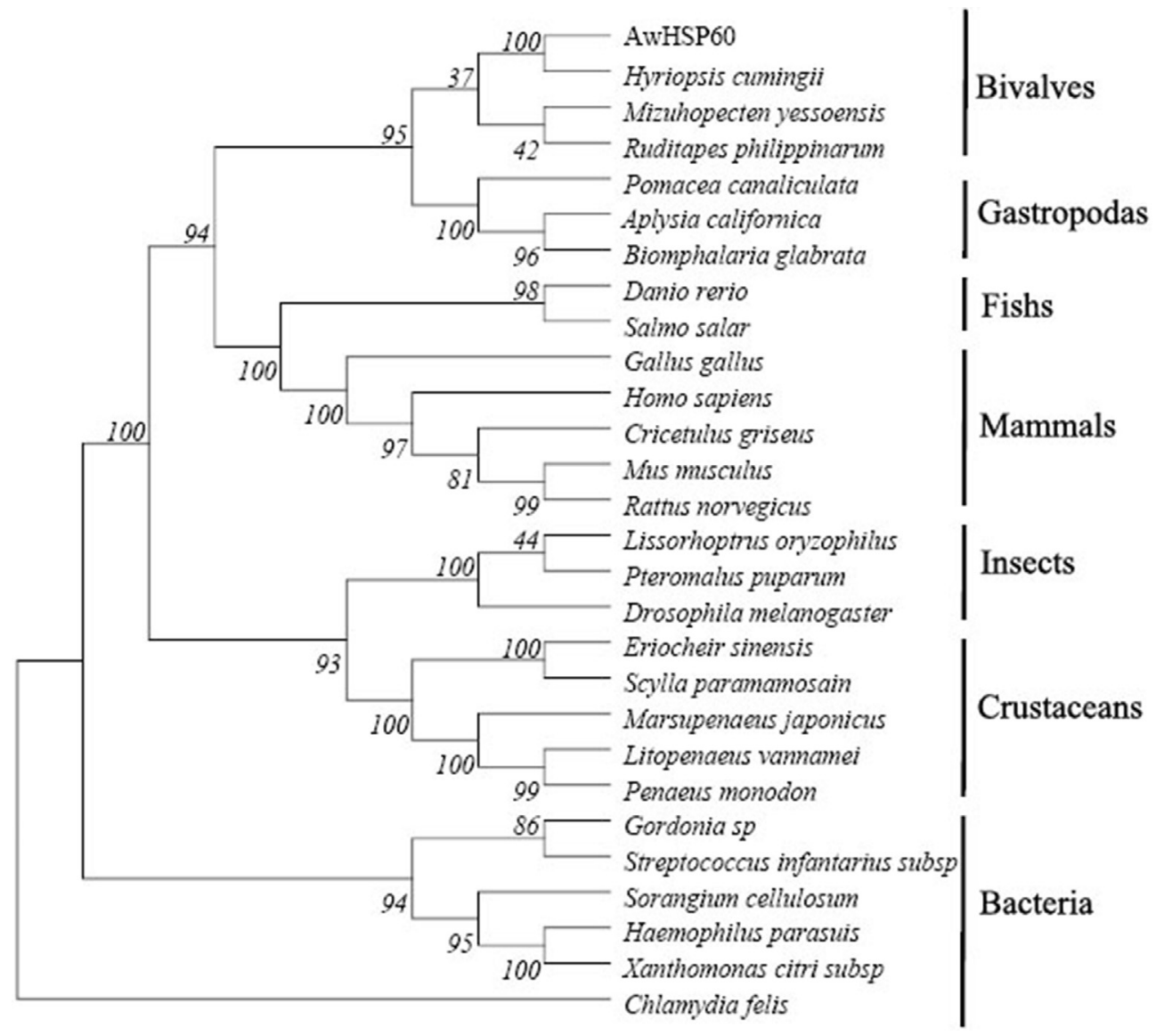

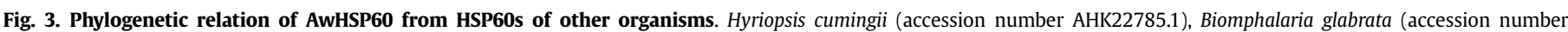

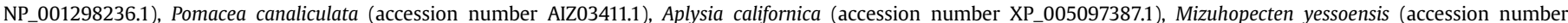

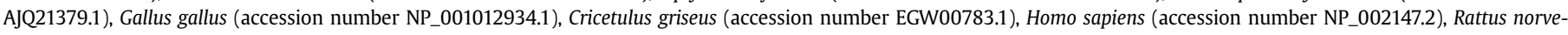

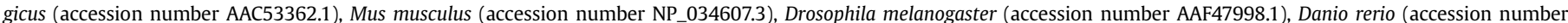

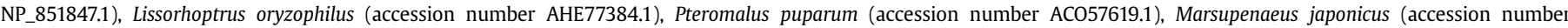

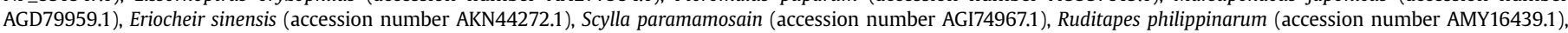

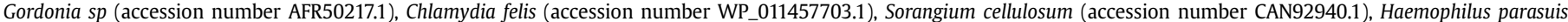

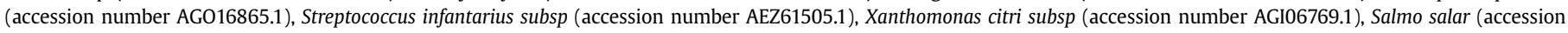
number ACN11370.1), Penaeus monodon (accession number ALS05377.1), Litopenaeus vannamei (accession number ACN30235.1). 


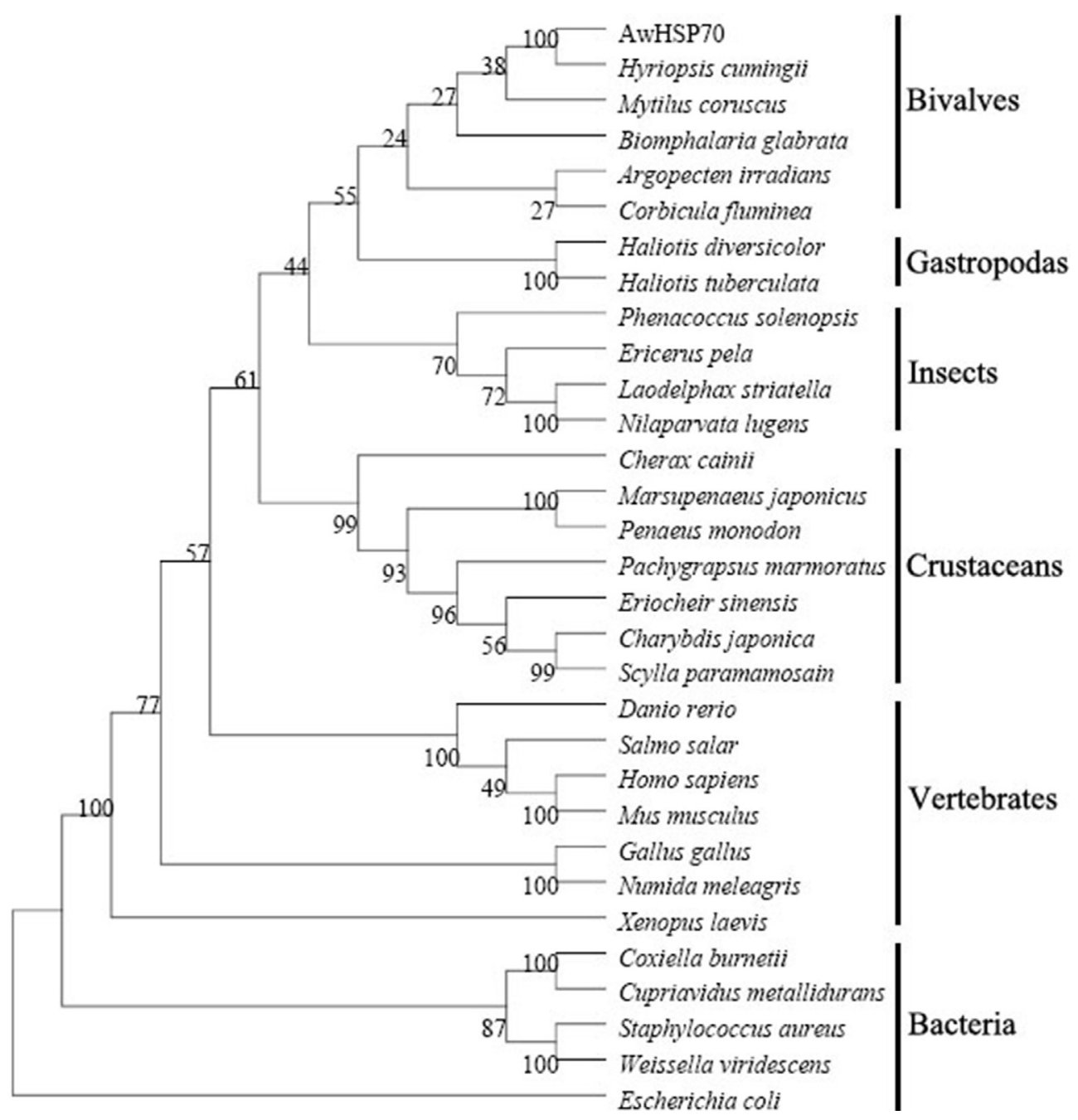

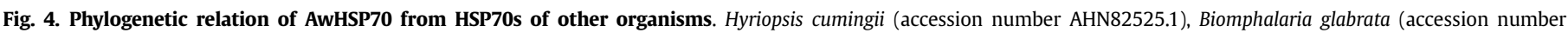

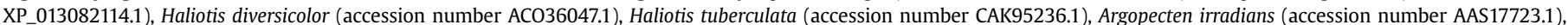

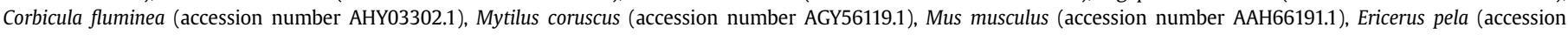

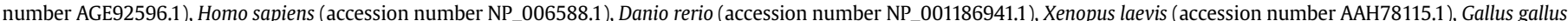

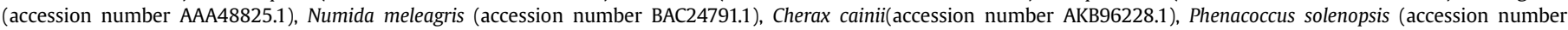

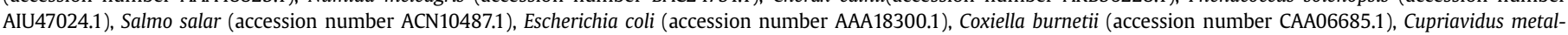

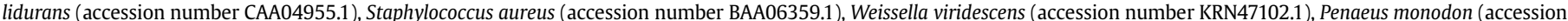

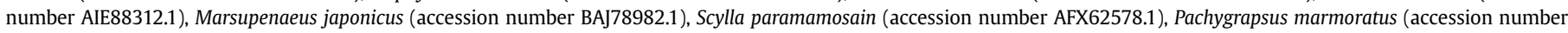

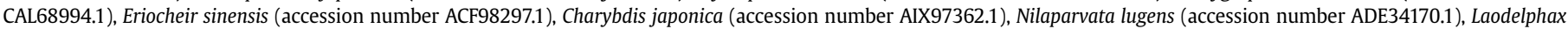
striatella (accession number AMD09926.1).

group, significant up-regulation of AwHSP70 expression showed a reversed $U$ shape characterized by an up-regulation from day $1-6$, a peak at day 6, a decline from day 6-15 (Fig. 7B).

In the hemocytes, AwHSP60 and AwHSP70 expressions of PBDE47 treated group respectively increased more 2.09 times $(P<0.05)$ and 1.81 times $(P<0.05)$ from day $1-15$ compared with that of control group (Fig. 8). In addition, up-regulation of AwHSP60 expression exhibited a time-dependent patter.

\section{Discussion}

Clams A. woodiana live in the sediment of ponds and filter suspended materials and colloids from large volumes of water. As such, it is a useful indicator for assessing the toxicity of water contaminants $[16,35]$. In the current study, A. woodiana was used to assess the effects of chronic exposure to PBDE-47 by evaluating transcript levels of AwHSP60 and AwHAP70 gene in the hepatopanceas, gills and hemocytes.

AwHSP60 had a continuous stretch of reading frame including ATP binding domains, transition regions and conserved domains, and share a high-degree similarity with that of mollusk, vertebrate, crustacean and insect. Most of pseudogenes generally show higher similarity with that of invertebrate, plants and microbe [36,37]. AwHSP60 protein showed an instability index of $29.23(<40)$ that indicated its high stability. With this regard, it precludes the assumption that AwHSP60 may be a pseudogene of prokaryotic origin. A conserved highly mitochondrial target sequence was fund in AwHSP60, suggested AwHSP60 is a mitochondrial chaperone according to earlier studies [38,39]. In the C-terminal region, AwHSP60 had a flexible GGM repeats which has been thought to play a specific role in facilitating the rearrangement of certain folding intermediate by providing a mildly hydrophobic and interactive surface $[40,41]$. In addition to ATP and $\mathrm{Mg}^{2+}$ binding charged domains, the apical to equatorial domain of AwHSP60 

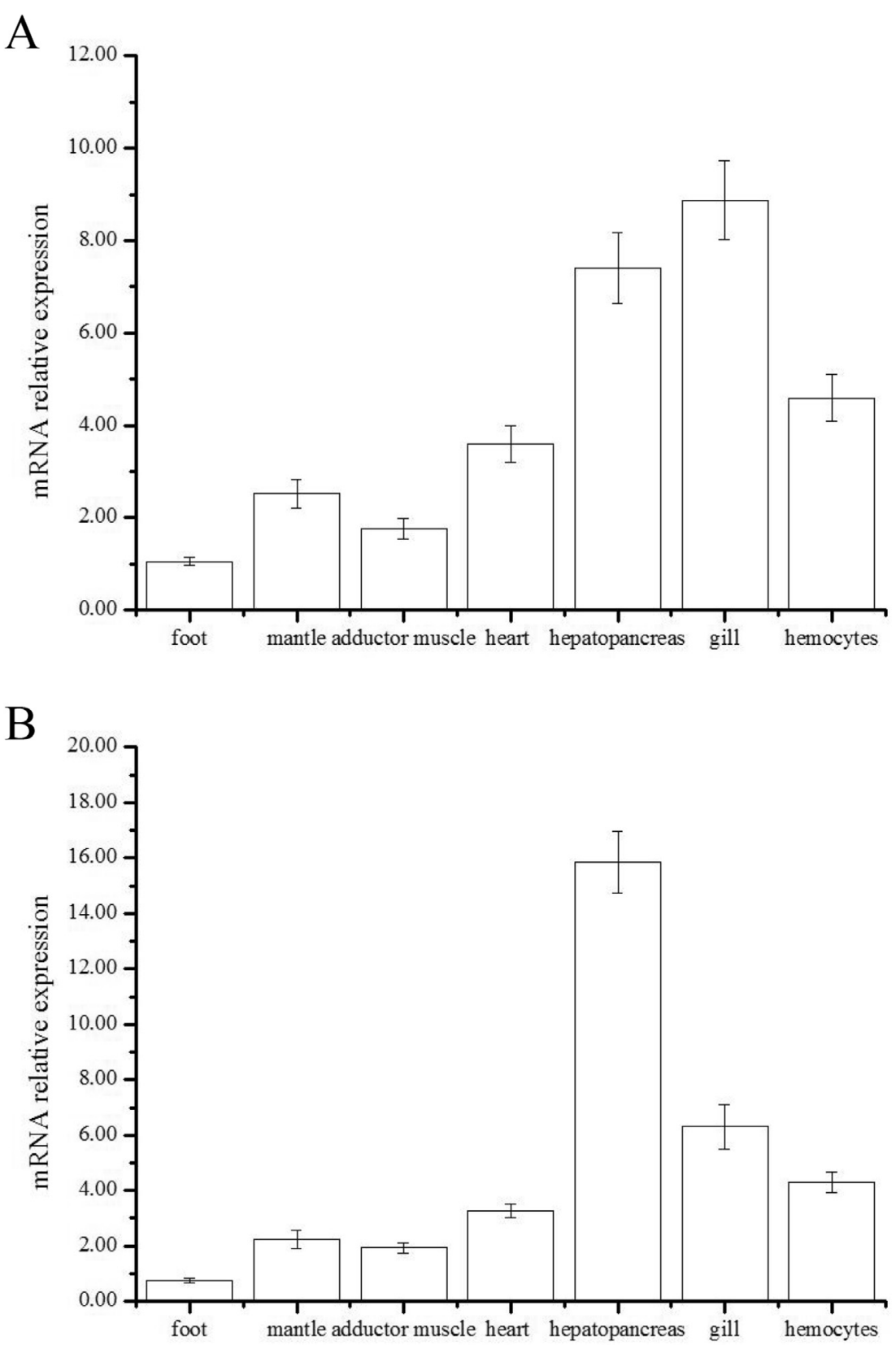

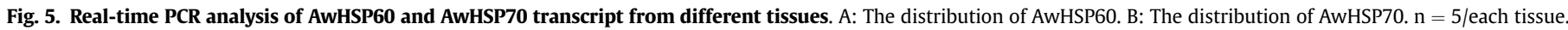

tertiary structures shows strong structural similarity with those of other species [40]. Based on aforementioned, AwHSP60 likely plays a potential role in folding of nascent or stress-denatured protein with respect of these highly conserved domains between AwHSP60 and members of HSP60 family.

Conserved domains and characteristic motifs of HSP70 family were observed in AwHSP70 protein sequence including the signatures 1, 2 and 3, the bipartite nuclear targeting sequence and ATP/ GTP-binding site motif A. In the region of C-terminus, the last four amino acids of EEVD formed specific motif of HSP70 family was fund in the AwHSP70, suggesting AwHSP70 is one of cytosolic HSP70s. HSP70 family are divided into different groups with respect of their sequence characterizations and distribution in the cells. Conserved domain of EEVD is regard as marker to distinguish cytosolic HSP70 from other groups. Notably, tetrapeptide motif of GGMP comprised consecutive repeats was fund in the AwHSP70, suggesting tetrapeptide motif of GGMP likely play an important role in formation of multi-chaperone complex as well as EEVD recognition. It is evidenced that formation of tetratrico-peptide repeat domain of HSPs is contribute to occurrence of multichaperone complex derived from HSP70, HSP90 and other cochaperones [42,43]. In addition, GGMP repeats is involved into formation of a structural entity together with the helical subdomain and the EEVD motif that functions as important player to mediate chaperone cofactor binding [44]. Notably, amino acid sequences of AwHSP70 share a significant similarity with that of other HSP70s, but nucleotide sequences of their UTRs are diverse. Similar phenomenon has also been reported in other studies. Therefore, we postulate that a complex mechanism is involved into transcription of HSP70 that is needed to elucidate in the future.

Combination of sequence alignment and phylogenetic analysis reveal HSP60 and HSP70 lineages are highly conserved from 
A

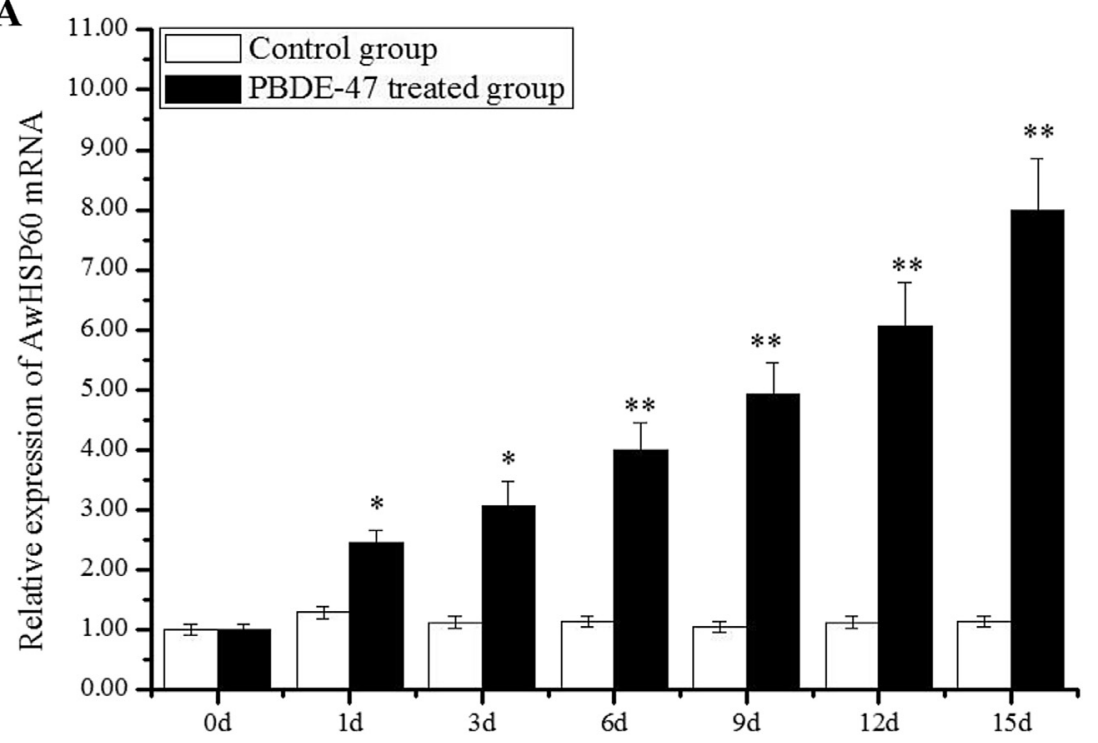

B

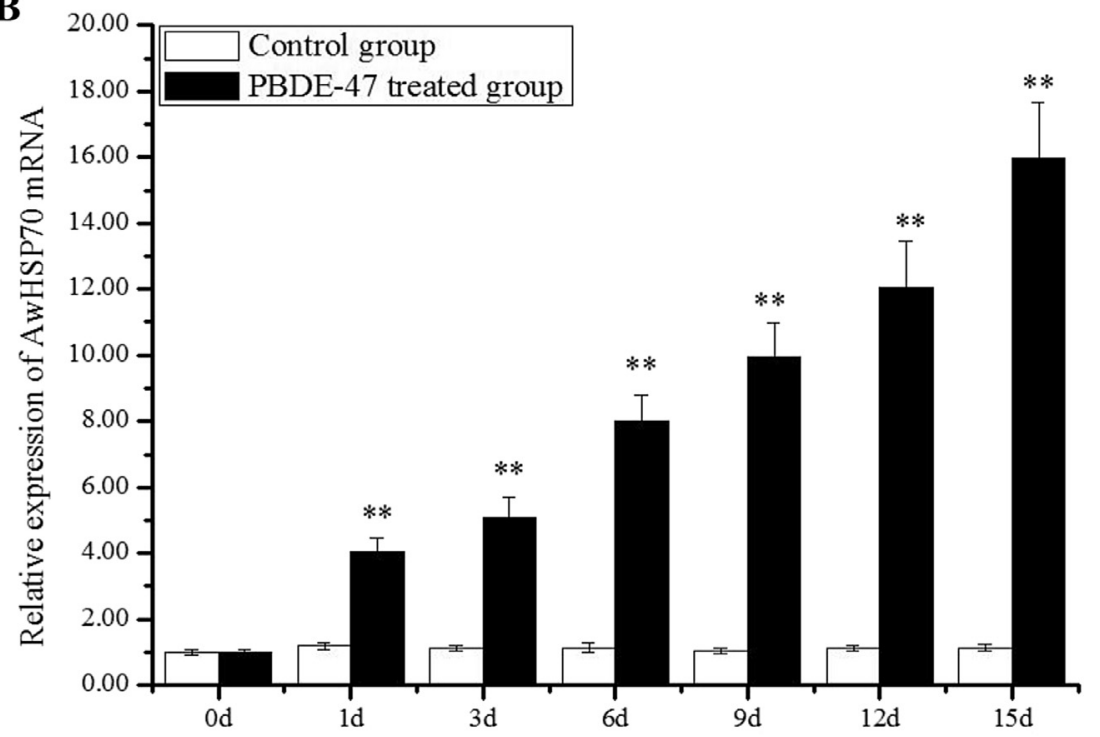

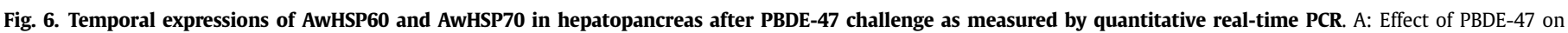

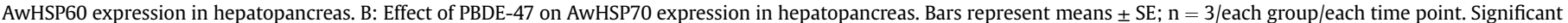
differences are performed by one-way ANOVA test. ${ }^{*} P<0.05,{ }^{* * *} P<0.01$ vs control group at the same time.

mollusk to insect and higher vertebrates. Meanwhile, it is also fund that freshwater bivalve $A$. woodiana and $H$. cumingii appear to be sibling relationship and come from a common ancestor. mRNA levels of AwHSP60 and AwHSP70 showed an ubiquitous distribution of bivalve A. woodiana that is associated adaption of AwHSP60 and AwHSP70 involved into. Meanwhile, expressions of AwHSP60 and AwHSP70 in hepatopancreas, gill and hemocytes showed a high profile. This distribution patter is likely associated with role of these organs. The hepatopancreas, a major organ for the digestion, absorption and secretory of compounds in mollusk, is sensitive to environmental changes [45]. Gill is the main entrance of contaminants present in the environment [46]. Bivalves have an open circulatory system populated by hemocytes that circulate in hemolymph vessels and sinuses as well as throughout soft tissues. Mucosal tissues are often well-irrigated by the hemocytes due to their primary role in exchanges with the surrounding environment for processes such as oxygen or nutrient extraction. Hemocytes is one important line of defense against environmental stress [16]. Hepatopancreas, gill and hemocytes play a key role in maintaining homeostasis of animals, also are targets acted by environmental factors $[16,45,46]$. So, higher expressions of AwHSP60 and AwHSP70 in these tissues are likely related with endurance of bivalve A. woodiana.

In the present study, administration of PBDE47 could result in a significant up-regulation of AwHSP60 and AwHSP70 expressions in hepatopancreas, gill and hemocytes compared with that of control group, suggest AwHSP60 and AwHSP70 play an important role in enhancing adaptation of $A$ woodiana exposed to PBDE-47 treatment. Clams $A$ woodiana are filter-feeding freshwater organism. Dissolved PBDEs of water are easy deposited and accumulated in cells in respect of their higher lipophilicity that can cause increase of ROS production. In normal condition, ROS will be rapidly 
A

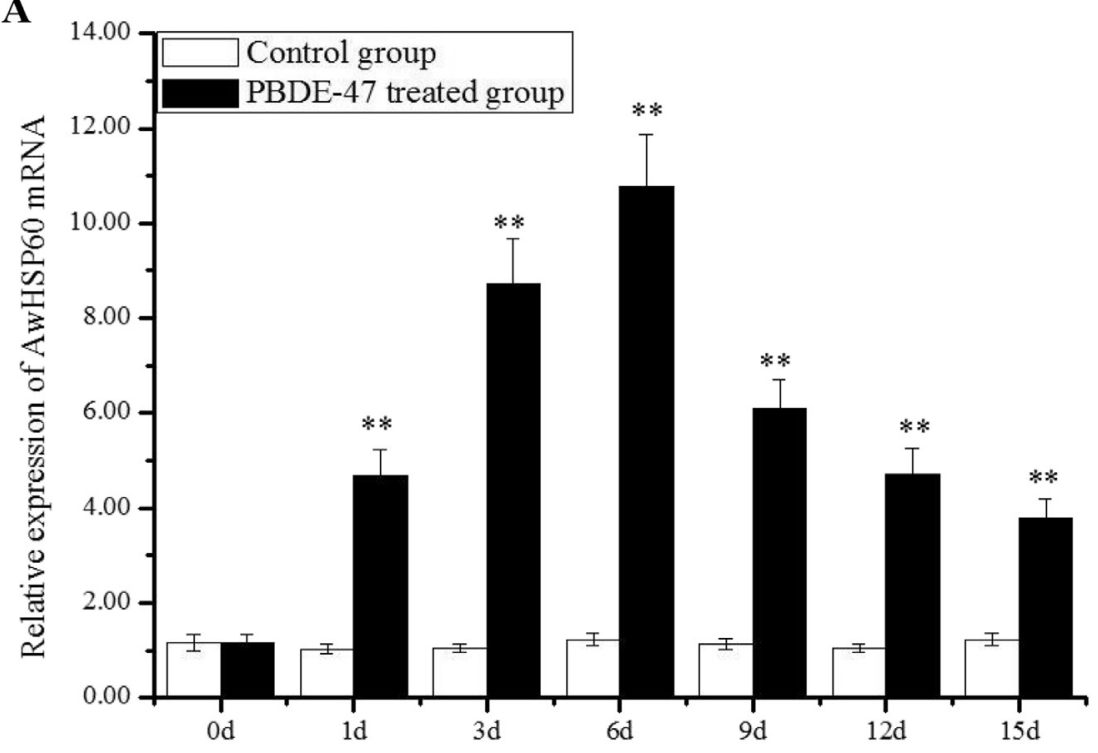

B

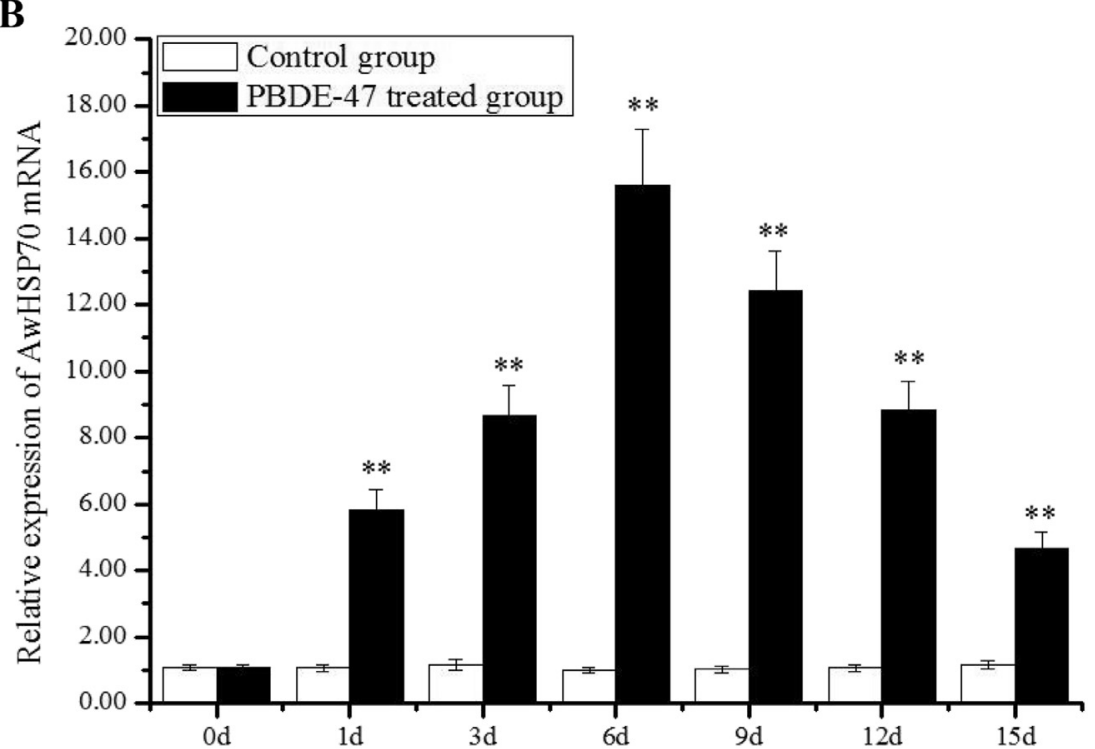

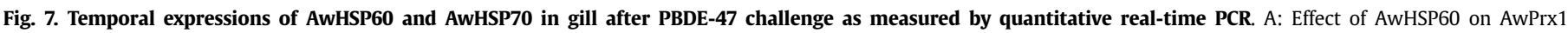

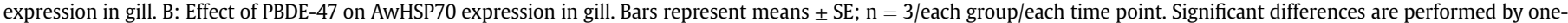
way ANOVA test. ${ }^{*} P<0.05,{ }^{* *} P<0.01$ vs control group at the same time.

scavenged and sustained at a rational level by an array of antioxidant enzymes. But, in the stress environment, production of higher ROS concentrations can lead to a risk of DNA damage, lipid peroxidation and protein carbonylation, that in turn results in an obvious increase of misfolded proteins. Up-regulation of HSPs is contributed to evaluating adaption by membrane translocation, degradation of misfolded proteins and other protein processing. Compared with others of HSPs, HSP60 and HSP70 are very widely studied and more sensitive to environmental stress. HSP70 expression is frequently used as a component of physiological mechanisms in the process mussels cope with environmental challenges. In mussel Mytilus coruscus, up-regulation of HSP70 expression exposed to heavy metals and organic hydro carbons is close related with the detoxification of these contaminants [47]. In the scallop Patinopecten yessoensis, expression of HSP70 shows an up-regulation matter under paralytic shellfish toxins stress that manifests an adaptive process to the toxic stress [48]. Additionally, induction of HSP70 expression is also observed in pacific oyster $C$. gigas challenged by high temperature that implies the involvement of HSP70s in response to thermal stress [49]. In some mussels, expression of HSP70 is activated by metals and it has regulatory pathways which are common to those of the family of metallothionein encoding genes [17-22]. Similarly, expression of HSP60 is up-regulated in the gills and digestive gland of oyster $C$. brasiliana after exposed to diesel fuel. mRNA levels of Hsp60 and Hsp70 are enhanced in the digestive gland of clam Corbicula fluminea after fluoxetine exposure [50]. An common pathway of environmental factors and pollutants interfering function of the body is to affect correct folding of native proteins in which activated HSPs expressions are key strategies to cope with damage (Sanders, 1993) [51]. Considering here, up-regulations of HSP70 and HSP60 are often considered as a general rule in aquatic organisms exposed to 
A
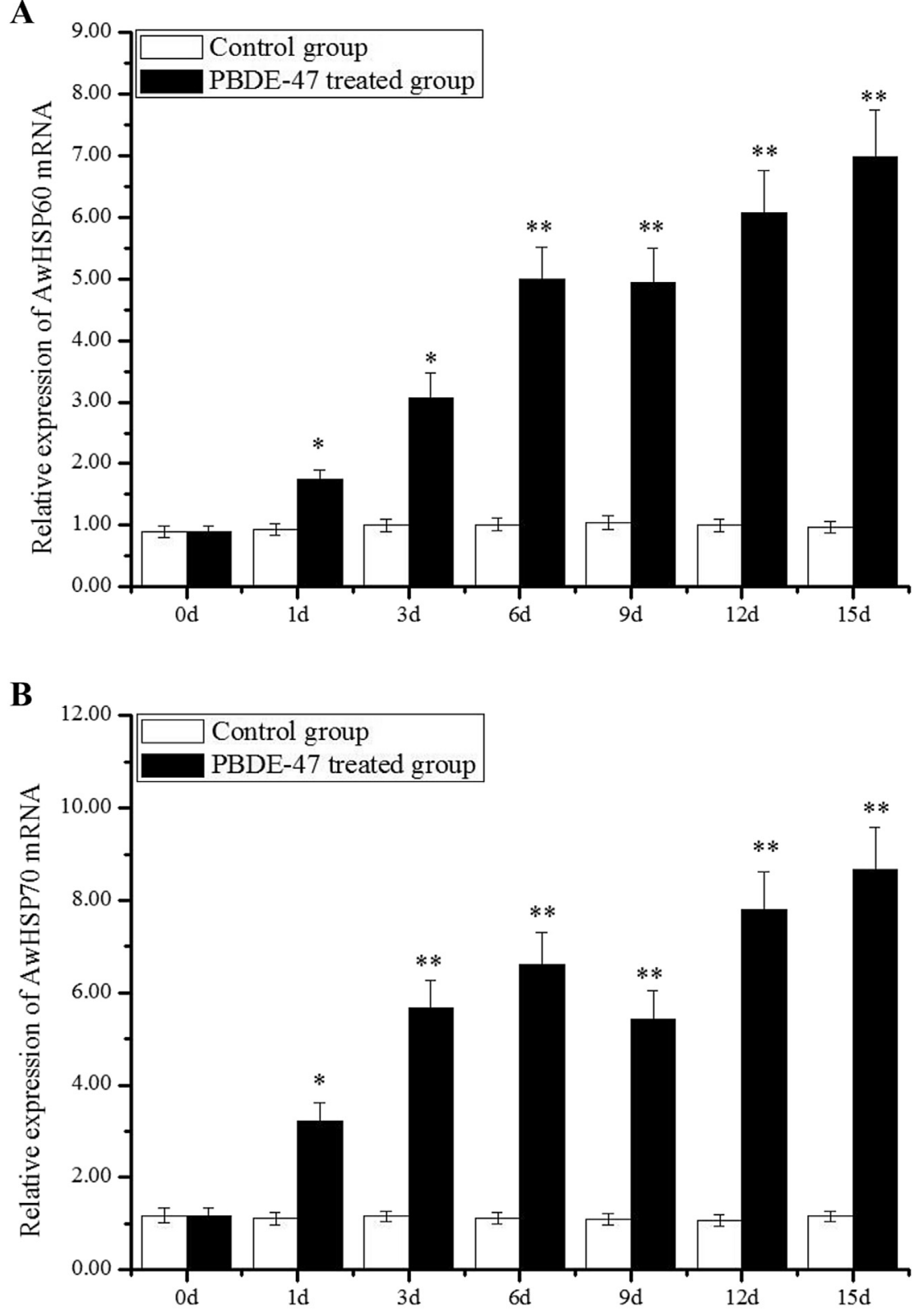

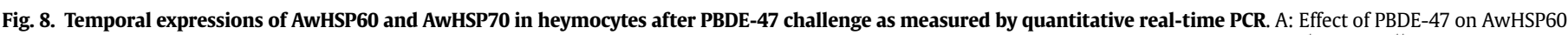

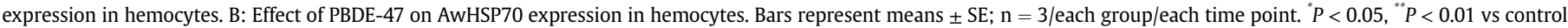
group at the same time. Significant differences are performed by one-way ANOVA test. ${ }^{\#} P<0.05$, ${ }^{\# \#} P<0.01$ vs control group at the day 0 .

environmental factors, heavy metal and pollutants. Therefore, upregulations of AwHSP60 and AwHSP70 expression work as an important player to against oxidative stress derived from PBDE-47.

In the hepatopancreas, up-regulations of AwHSP70 and AwHSP60 mRNA level respectively showed a time-dependent pattern that is likely related with compensation mechanism of organism. Under prolonged exposure, animals have a stress management through an adaptive response, in which they probably need for restoration of cell homeostasis. As an intracellular chaperone, HSP70 is involved into extracellular immunoregulatory functions and protect cells against environmental stress in which it often exhibit a tissue-/time-/dose-dependent expression matter $[9,10]$. In the hepatopancreas of $M$. galloprovincialis, timedependent mRNA expressions of HSP70 are found in heavy metals and other chemicals treatment, such as copper, cadmium and 180 CST fuel [20]. In the scallop Patinopecten yessoensis, upregulation of HSP70 expressions also displays a time dependent pattern in response to toxic dinoflagellate exposure [48]. In addition, time-dependent expression pattern of HSP70 is also observed in C. gigas from high temperature response [49]. Furthermore, mRNA levels of HSP70 and HSP90 are induced by benzo(a)pyrene and the absolute expression levels of them show a temporal and dose-dependent response in the digestive gland of Ruditapes philippinarum [8].

During whole experiment observed, expressions of AwHSP60 and AwHSP70 in the gill showed a reversed U profile characterized by an up-regulation at early stage, a peak at middle stage and a decline at latter stage that is in line with the hormesis phenomenon in toxicology. This phenomenon is likely associated with endurance ability of animals faced persistent production of oxidative stress. 
With elongation of PBDE-47 treated time, ROS should gradually be generated that cause more oxidative stress on the cells because of constantly deposition of PBDE-47 in body. On another hand, strategy of up-regulating HSPs expressions is employed to handle disfolded proteins in order to enhancing endurance [52]. Once level of oxidative stress derived from PBDE-47 treatment reaches to a peak and exceeds the endurance ability of cells, tremendous stress should result in a chronic injury, inflammation and apoptosis of immune cells [53,54]. Under this condition, if animals lack enough power to produce new cells to compensate for death ones, quantity of living cells should be gradually decrease [55,56]. Following, expressions of AwHSP60 and AwHSP70 are also decline at latter stage of PBDE-47 treatment.

Noteworthy, expressions of AwHSP60 and AwHSP70 respectively showed different spatial and temporal characterizations in the hapetoparaces, gill and hemocytes, suggest AwHSP60 and AwHSP70 are likely involved into different regulatory routes to cope with oxidative stress. Hepatopancreas of the molluscs show double properties of combination liver and pancreas, is involved into digestion and neutralizes large amounts of toxicants [45]. Gill is directly interact with the outer environment and the main entrance of contaminants present in the environment [46]. Hemocytes of mussels is the important line of defense against pathogenic invade as well as reducing ROS through phagocytosis and stimulation of respiratory burst, and widely used as hallmark in environmental toxicology [16]. Therefore, in order to sustain normal function of organs, AwHSP60 and AwHSP70 are likely take part in different pathway to cope with environmental stress in these tissues. Meanwhile, like previous reports, induction of HSPs is a complex event.

In conclusion, cDNAs of AwHSP60 and AwHSP70 were firstly cloned from bivalve $A$. woodiana and their full-length cDNA respectively contained highly conserved motifs and residues. Treatment of PBDE-47 could result in a significant up-regulation of AwHSP60 and AwHSP70 expressions in the hepatoparances, gills and hemocytes. In addition, expressions of AwHSP60 and AwHSP70 show different characterizations to deal with oxidative stress along elongation of PBDE-47 treated time. Correlating the tissue specific mRNA expressions and transcriptional inductions of AwHSP60 and AwHSP70 by PBDE-47 suggests that $A$. woodiana may work as a bioindicator for assessing the impact of freshwater pollutants with the application of gene expression concept. On the other hand, government should concern PBDEs diffusion and take measures to prevent or mitigate extensive negative impacts on freshwater organisms and conserve aquatic organism biodiversity.

\section{Acknowledgement}

This research was funded by the National Natural Science Foundation of Henan (No.2015GGJS-286, 17A180010) and China Postdoctoral Science Foundation Funded Project (2016M590143).

\section{References}

[1] M.G. Santoro, Heat shock factors and the control of the stress response, Biochem. Pharmacol. 59 (2000) 55-63.

[2] F.U. Hartl, M. Hayer-Hartl, Molecular chaperones in the cytosol: from nascent chain to folded protein, Science 295 (2002) 1852-1858.

[3] K.C. Kregel, Heat shock proteins: modifying factors in physiological stress responses and acquired thermotolerance, J. Appl. Physiol. 92 (2002) (1985) $2177-2186$

[4] J.C. Young, Mechanisms of the Hsp70 chaperone system, Biochem. Cell Biol. 88 (2010) 291-300

[5] A.L. Joly, G. Wettstein, G. Mignot, F. Ghiringhelli, C. Garrido, Dual role of heat shock proteins as regulators of apoptosis and innate immunity, J. Innate Immun. 2 (2010) 238-247.

[6] M.P. Mayer, B. Bukau, Hsp70 chaperones: cellular functions and molecular mechanism, Cell Mol. Life Sci. 62 (2005) 670-684.
[7] H.B. Li, Y.Z. Du, Molecular cloning and characterization of an Hsp90/70 organizing protein gene from Frankliniella occidentalis (Insecta: Thysanoptera, Thripidae), Gene 520 (2013) 148-155.

[8] T. Liu, L. Pan, Y. Cai, J. Miao, Molecular cloning and sequence analysis of heat shock proteins 70 (HSP70) and 90 (HSP90) and their expression analysis when exposed to benzo(a)pyrene in the clam Ruditapes philippinarum, Gene 555 (2015) 108-118.

[9] Y. Xu, G. Zheng, S. Dong, G. Liu, X. Yu, Molecular cloning, characterization and expression analysis of HSP60, HSP70 and HSP90 in the golden apple snail, Pomacea canaliculata, Fish. Shellfish Immunol. 41 (2014) 643-653.

[10] S. Krenek, M. Schlegel, T.U. Berendonk, Convergent evolution of heatinducibility during subfunctionalization of the Hsp70 gene family, BMC Evol. Biol. 13 (2013) 1-15.

[11] H.H. Liu, J.Y. He, C.F. Chi, J. Shao, Differential HSP70 expression in Mytilus coruscus under various stressors, Gene 543 (2014) 166-173.

[12] S. Chen, A.M. Roseman, A.S. Hunter, S.P. Wood, S.G. Burston, N.A. Ranson, A.R. Clarke, H.R. Saibil, Location of a folding protein and shape changes in GroEL-GroES complexes imaged by cryo-electron microscopy, Nature 371 (1994) 261-264.

[13] Z. Xu, A.L. Horwich, P.B. Sigler, The crystal structure of the asymmetric GroELGroES-(ADP)7 chaperonin complex, Nature 388 (1997) 741-750.

[14] D.K. Clare, D. Vasishtan, S. Stagg, J. Quispe, G.W. Farr, M. Topf, A.L. Horwich, H.R. Saibil, ATP-triggered conformational changes delineate substrate-binding and -folding mechanics of the GroEL chaperonin, Cell 149 (2012) 113-123.

[15] B. Allam, E. Pales Espinosa, Bivalve immunity and response to infections: are we looking at the right place? Fish. Shellfish Immunol. 53 (2016) 4-12.

[16] X. Guo, Y. He, L. Zhang, C. Lelong, A. Jouaux, Immune and stress responses in oysters with insights on adaptation, Fish. Shellfish Immunol. 46 (2015) $107-119$.

[17] I. Gourdon, L. Gricourt, K. Kellner, P. Roch, J.M. Escoubas, Characterization of a cDNA encoding a $72 \mathrm{kDa}$ heat shock cognate protein (Hsc72) from the Pacific oyster, Crassostrea gigas, DNA Seq. 11 (2000) 265-270.

[18] A.V. Rathinam, T.T. Chen, R.M. Grossfeld, Cloning and sequence analysis of a cDNA for an inducible $70 \mathrm{kDa}$ heat shock protein (Hsp70) of the American oyster (Crassostrea virginica), DNA Seq. 11 (2000) 261-264.

[19] A. Piano, P. Valbonesi, E. Fabbri, Expression of cytoprotective proteins, heat shock protein 70 and metallothioneins, in tissues of Ostrea edulis exposed to heat and heavy metals, Cell Stress Chaperones 9 (2004) 134-142.

[20] S. Franzellitti, E. Fabbri, Differential HSP70 gene expression in the Mediterranean mussel exposed to various stressors, Biochem. Biophys. Res. Commun. 336 (2005) 1157-1163.

[21] L. Song, L. Wu, D. Ni, Y. Chang, W. Xu, K. Xing, The cDNA cloning and mRNA expression of heat shock protein 70 gene in the haemocytes of bay scallop (Argopecten irradians, Lamarck 1819) responding to bacteria challenge and naphthalin stress, Fish. Shellfish Immunol. 21 (2006) 335-345.

[22] X. Yue, B. Liu, L. Sun, B. Tang, Cloning and characterization of a hsp70 gene from Asiatic hard clam Meretrix meretrix which is involved in the immune response against bacterial infection, Fish. Shellfish Immunol. 30 (2011) 791-799.

[23] M. Soroka, Genetic variability among freshwater mussel Anodonta woodiana (Lea, 1834) (Bivalvia: Unionidae) populations recently introduced in Poland, Zool. Sci. 22 (2005) 1137-1144.

[24] S. Kolarević, J. Knežević-Vukčević, M. Paunović, M. Kračun, B. Vasiljević, J. Tomović, B. Vuković-Gačić, Z. Gačić, Monitoring of DNA damage in haemocytes of freshwater mussel Sinanodonta woodiana sampled from the Velika Morava River in Serbia with the comet assay, Chemosphere 93 (2013) 243-251.

[25] M. Reichard, K. Douda, M. Przybyłski, O.P. Popa, E. Karbanová, K. Matasová, K. Rylková, M. Polačik, R. Blažek, C. Smith, Population-specific responses to an invasive species, Proc. Biol. Sci. 282 (2015) 20151063.

[26] H. Liu, J. Yang, J. Gan, Trace element accumulation in bivalve mussels Anodonta woodiana from Taihu Lake, China, Arch. Environ. Contam. Toxicol. 59 (2010) 593-601.

[27] S. Uno, H. Shiraishi, S. Hatakeyama, A. Otsuki, J. Koyama, Accumulative characteristics of pesticide residues in organs of bivalves (Anodonta woodiana and Corbicula leana) under natural conditions, Arch. Environ. Contam. Toxicol. 40 (2001) 35-48.

[28] J. Yang, H. Harino, H. Liu, N. Miyazaki, Monitoring the organotin contamination in the Taihu Lake of China by bivalve mussel Anodonta woodiana, Bull. Environ. Contam. Toxicol. 81 (2008) 164-168.

[29] J.T. Hartmann, S. Beggel, K. Auerswald, B.C. Stoeckle, J. Geist, Establishing mussel behavior as a biomarker in ecotoxicology, Aquat. Toxicol. 170 (2015) 279-288.

[30] C.T. Callil, D. Krinski, F.A. Silva, Variations on the larval incubation of Anodontites trapesialis (Unionoida, Mycetopodidae): synergetic effect of the environmental factors and host availability, Braz J. Biol. 72 (2012) 545-552.

[31] A. Zieritz, B. Gum, R. Kuehn, J. Geist, Identifying freshwater mussels (Unionoida) and parasitic glochidia larvae from host fish gills: a molecular key to the North and Central European species, Ecol. Evol. 2 (2012) 740-750.

[32] P. Berton, N.B. Lana, J.M. Ríos, J.F. García-Reyes, J.C. Altamirano, State of the art of environmentally friendly sample preparation approaches for determination of PBDEs and metabolites in environmental and biological samples: a critical review, Anal. Chim. Acta 905 (2016) 24-41.

[33] X. Xia, C. Huang, D. Zhang, Y. Zhang, S. Xue, X. Wang, Q. Zhang, L. Guo, Molecular cloning, characterization, and the response of $\mathrm{Cu} / \mathrm{Zn}$ superoxide 
dismutase and catalase to PBDE-47 and -209 from the freshwater bivalve Anodonta woodiana, Fish. Shellfish Immunol. 51 (2016) 200-210.

[34] X. Xia, C. Hua, S. Xue, B. Shi, G. Gui, D. Zhang, X. Wang, L. Guo, Response of selenium-dependent glutathione peroxidase in the freshwater bivalve Anodonta woodiana exposed to 2,4-dichlorophenol,2,4,6-trichlorophenol and pentachlorophenol, Fish. Shellfish Immunol. 55 (2016) 499-509.

[35] L. Canesi, C. Ciacci, R. Fabbri, A. Marcomini, G. Pojana, G. Gallo, Bivalve molluscs as a unique target group for nanoparticle toxicity, Mar. Environ. Res. 76 (2012) 16-21.

[36] L. Li, B. Zhao, Z. Cui, J. Gan, M.K. Sakharkar, P. Kangueane, Identification of hot spot residues at protein-protein interface, Bioinformation 1 (2006) 121-126.

[37] J.E. Hill, A. Paccagnella, K. Law, P.L. Melito, D.L. Woodward, L. Price, A.H. Leung L.K. Ng, S.M. Hemmingsen, S.H. Goh, Identification of Campylobacter spp. and discrimination from Helicobacter and Arcobacter spp. by direct sequencing of PCR-amplified cpn60 sequences and comparison to cpnDB, a chaperonin reference sequence database, J. Med. Microbiol. 55 (2006) 393-399.

[38] J.E. Hill, S.L. Penny, K.G. Crowell, S.H. Goh, S.M. Hemmingsen, cpnDB: chaperonin sequence database, Genome Res. 14 (2004) 1669-1675.

[39] J. Deng, M. Yang, Y. Chen, X. Chen, J. Liu, S. Sun, H. Cheng, Y. Li, E.H. Bigio, M. Mesulam, Q. Xu, S. Du, K. Fushimi, L. Zhu, J.Y. Wu, FUS Interacts with HSP60 to promote mitochondrial damage, PLoS Genet. 11 (2015) e1005357.

[40] M. Kumar Singh, P.V. Janardhan Reddy, A.S. Sreedhar, P.K. Tiwari, Molecular characterization and expression analysis of hsp60 gene homologue of sheep blowfly, Lucilia cuprina, J. Therm. Biol. 52 (2015) 24-37.

[41] Y.C. Tang, H.C. Chang, A. Roeben, D. Wischnewski, N. Wischnewski, M.J. Kerner, F.U. Hartl, M. Hayer-Hartl, Structural features of the GroEL-GroES nano-cage required for rapid folding of encapsulated protein, Cell 125 (2006) 903-914.

[42] B.C. Freeman, M.P. Myers, R. Schumacher, R. Morimoto, Identification of a regulatory motif in Hsp70 that affects ATPase activity, substrate binding and interaction with Hdj-1, EMBO J. 14 (1995) 2281-2292.

[43] C. Scheufler, A. Brinker, G. Bourenkov, S. Pegoraro, L. Moroder, H. Bartunik, F.U. Hartl, I. Moarefi, Structure of TPR domain-peptide complexes: critica elements in the assembly of the Hsp70-Hsp90 multichaperone machine, Cell 101 (2000) 199-210.

[44] J. Demand, J. Luders, J. Hohfeld, The carboxy-terminal domain of Hsc70 provides binding sites for a distinct set of chaperone cofactors, Mol. Cell. Biol. 18 (1998) 2023-2028.
[45] Y. Irnidayanti, Toxicity and traces of $\mathrm{Hg}, \mathrm{Pb}$ and $\mathrm{Cd}$ in the hepatopancreas, gills and muscles of Perna viridis from Jakarta Bay, Indonesia, Pak J. Biol. Sci. 18 (2015) 94-98.

[46] R.S. Piazza, R. Trevisan, F. Flores-Nunes, G. Toledo-Silva, N. Wendt, J.J. Mattos, D. Lima, S. Taniguchi, S.T. Sasaki, Exposure to phenanthrene and depuration: changes on gene transcription, enzymatic activity and lipid peroxidation in gill of scallops Nodipecten nodosus, Aquat. Toxicol. 177 (2016) 146-155.

[47] V.L. Maria, M.J. Bebianno, Antioxidant and lipid peroxidation responses in Mytilus galloprovincialis exposed to mixtures of benzo(a)pyrene and copper, Comp. Biochem. Physiol. C Toxicol. Pharmacol. 154 (2011) 56-63.

[48] J. Cheng, X. Xun, Y. Kong, S. Wang, Z. Yang, Y. Li, D. Kong, S. Wang, L. Zhang, X. Hu, Z. Bao, Hsp70 gene expansions in the scallop Patinopecten yessoensis and their expression regulation after exposure to the toxic dinoflagellate Alexandrium catenella, Fish. Shellfish Immunol. 58 (2016) 266-273.

[49] Q. Zhu, L. Zhang, L. Li, H. Que, G. Zhang, Expression Characterization of stress genes under high and low temperature stresses in the Pacific oyster, Crassostrea gigas, Mar. Biotechnol. (NY) 18 (2016) 176-188.

[50] H. Chen, J. Zha, X. Liang, J. Bu, M. Wang, Z. Wang, Sequencing and de novo assembly of the Asian clam (Corbicula fluminea) transcriptome using the Illumina GAIIx method, PLoS One 8 (2013) e79516.

[51] B.M. Sanders, Stress proteins in aquatic organisms: an environmental perspective, Crit. Rev. Toxicol. 23 (1993) 49-75.

[52] S.A. Lozano-Sepulveda, O.L. Bryan-Marrugo, C. Cordova-Fletes, M.C. GutierrezRuiz, A.M. Rivas-Estilla, Oxidative stress modulation in hepatitis C virus infected cells, World J. Hepatol. 7 (2015) 2880-2889.

[53] E.J. Calabrese, U-shaped dose response in behavioral pharmacology: historical foundations, Crit. Rev. Toxicol. 38 (2008) 591-598.

[54] A.A. Soldatov, O.L. Gostyukhina, I.V. Golovina, Antioxidant enzyme complex of tissues of the bivalve Mytilus galloprovincialis Lam. under normal and oxidative-stress conditions: a review, Prikl. Biokhim Mikrobiol. 43 (2007) $621-628$.

[55] R.K. Pipe, J.A. Coles, F.M.M. Carissan, K. Ramanathan, Copper induced immunomodulation in the marine mussel, Mytilus edulis, Aquat. Toxicol. 46 (1999) 43-54.

[56] M. De Zoysa, W.A. Pushpamali, C. Oh, I. Whang, S.J. Kim, J. Lee, Transcriptional up-regulation of disk abalone selenium dependent glutathione peroxidase by $\mathrm{H}_{2} \mathrm{O}_{2}$ oxidative stress and Vibrio alginolyticus bacterial infection, Fish Shellfish Immunol. 25 (2008) 446-457. 